\title{
MODELING SNOW CRYSTAL GROWTH I: Rigorous Results for Packard's Digital Snowflakes
}

\author{
JANKO GRAVNER \\ Mathematics Department \\ University of California \\ Davis, CA 95616 \\ e-mail: gravner@math.ucdavis.edu \\ DAVID GRIFFEATH \\ Department of Mathematics \\ University of Wisconsin \\ Madison, WI 53706 \\ e-mail: griffeat@math.wisc.edu
}

(Final version, October 2006)

\begin{abstract}
Digital snowflakes are solidifying cellular automata on the triangular lattice with the property that a site having exactly one occupied neighbor always becomes occupied at the next time. We demonstrate that each such rule fills the lattice with an asymptotic density that is independent of the initial finite set. There are some cases in which this density can be computed exactly, and others in which it can only be approximated. We also characterize when the final occupied set comes within a uniformly bounded distance of every lattice point. Other issues addressed include macroscopic dynamics and exact solvability.
\end{abstract}

2000 Mathematics Subject Classification. Primary 37B15. Secondary 68Q80, 11B05, 60K05.

Keywords: asymptotic density, cellular automaton, exact solvability, growth model, macroscopic dynamics, thickness.

Acknowledgments. We thank Ken Libbrecht for sharing with us his unmatched expertise on real snowflakes, and Mirek Wójtowicz for developing the MCell cellular automata explorer. We are also grateful to Dean Hickerson who pointed out that the rules Hex 14, Hex 145, Hex 146, and Hex 1456, started from a singleton, all generate different final sets.

Support. JG was partially supported by NSF grant DMS-0204376 and the Republic of Slovenia's Ministry of Science program P1-285. DG was partially supported by NSF grant DMS0204018.

\section{Introduction}

Six-sided ice crystals that fall to earth in ideal winter conditions, commonly known as snowflakes, have fascinated scientists for centuries. They exhibit a seemingly endless variety of shape and structure, often dendritic and strangely botanical, yet highly symmetric and mathematical in 
their designs. To this day, snowflake growth from molecular scales, with its tension between disorder and pattern formation, remains mysterious in many respects.

Study of snowflakes dates back at least to the 16th century [Mag], and includes early contributions from such scientific giants as Kepler [Kep], Hooke [Hoo], and Descartes [Des]. With the advent of cameras came the first snow crystal album: more than 5,000 photos collected by W. Bentley beginning in 1885 (see $[\mathrm{BH}]$ ). Although rather few people have ever seen such crystals with their own eyes, Bentley's images helped establish snowflake designs, simplified and idealized, as universal icons for wintertime. The most significant scientific advances of the past century were due to Nakaya [Nak] in the 1930s, who classified natural crystals into dozens of types, first grew synthetic crystals in the laboratory, and discovered an elaborate, still perplexing morphology diagram, which predicts the predominant type of snowflake arising at any given temperature and supersaturation level. (In particular, the familiar, essentially two-dimensional crystals arise only for certain parameter values; in other conditions columnar "needles" form.) All this and much more is explained superbly in a recent popular account by Libbrecht and Rasmussen [LR], which also contains a great many state-of-the-art photographs of breathtaking beauty. There is a companion web site [Lib1]; see also [Lib2] for a current scholarly review.

Over the past century, geometric structures inspired by snow crystals have begun to adorn the world of mathematics. Most celebrated is the Koch snowflake [Kin], introduced by H. von Koch [Koc] in 1904. One of the earliest known fractals, this closed curve with Hausdorff dimension $2 \log 2 / \log 3$ is obtained in the limit by starting from an equilateral triangle (with $\bullet$ markers at its vertices) and repeatedly applying the substitution scheme

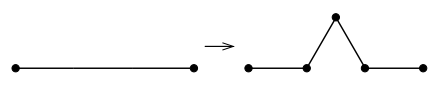

to each piece between markers. More recent variations on Koch's construction include Gosper's flowsnake [Gar] and the pentaflake [Dix]. While none of these designs resembles a real snow crystal to any great extent, their blend of elementary polygonal shapes with infinitely fine branching detail evokes the same iconography as Bentley's album.

The building blocks for snowflakes are hexagonally arranged molecules of natural ice $(I h)$. Just how the elaborate designs emerge as water vapor freezes is still poorly understood. Only very recently have a few rudimentary movies of synthetic crystal growth been produced (cf. [LR, p. 57]). The solidification process involves complex physical chemistry of diffusion limited aggregation and attachment kinetics. Theoretical research and mathematical modeling to date have mainly focused on the evolution of dendrite tips. See [Gle, pp. 309-314] for a popular account of the challenges, $[\mathrm{Mea}]$ and $[\mathrm{PV}]$ for recent scholarly monographs, and [Ada] for a current review.

In 1984, Packard [Pac] introduced a supremely simple cellular automaton (CA) model for crystal solidification. On a honeycomb lattice of hexagonal cells, start with a single "seed" cell of ice surrounded by vapor. At each subsequent discrete-time update, any vapor cell neighboring the requisite number of frozen cells turns to ice. Since real snowflake growth favors the tips of the crystal, Packard proposed that exactly one occupied (frozen) neighbor should cause solidification, but exactly two should not. Thus, in one of his digital snowflakes a site joins the crystal if and only if it has exactly one occupied neighbor, while in another it joins if the number of occupied neighbors is odd. In the present paper we will refer to these rules as Hex 1 and Hex 135, respectively. 
Packard's snowflake automata have been widely publicized since the 1980s to illustrate how very simple mathematical algorithms can emulate complex natural phenomena. A multicolor image of Hex 135 occupies nearly all of p. 189 in Wolfram's 1984 article [Wol1], and the same graphic is reproduced as the first color plate of Steven Levy's 1992 book [Lev]. More recently, the first 30 updates of Hex 1 are illustrated on p. 371 of [Wol3]. The central tenet of [Wol1], already familiar from the established universality of Conway's Game of Life [BCG], was that "Simulation by computer may be the only way to predict how certain complicated systems evolve." Implicit in this perspective is the inadequacy of mathematics to analyze complexity. In the discussion of Hex 135 and the corresponding caption, he writes:

"Snowflakes grown in a computer experiment from a single frozen cell according to this rule show intricate treelike patterns, which bear a close resemblance to real snowflakes. [...] The only practical way to generate the pattern is by computer simulation."

Levy's account reiterates the claimed verisimilitude:

"An elementary schoolchild could look at any of the gorgeous pictures of computer screens in Packard's collection and instantly identify it as a snowflake."

So how do these digital snowflakes evolve? The left frame of Fig. 1 shows a representative snapshot of Hex 134 after 218 updates starting from a single occupied cell. (The graphic in [Wol1] and [Lev] is quite similar.) Letting $A_{t}$ denote the crystal at time $t$, started from $A_{0}=\{0\}$, it turns out that $A_{2^{n}}$ occupies, with a certain density, the hexagon of lattice cells within $2^{n}$ steps of the origin for each $n$, but that shapes with apparently fractal boundary arise in the limit along intermediate subsequences of the form $t_{n}=\left\lfloor a 2^{n}\right\rfloor$ when $a$ is not a dyadic rational. For instance, the limit shape for Hex 1 and Hex 135 along the $a=1 / 3$ subsequence is exactly the Koch-type snowflake starting from a regular hexagon (with $\bullet$ markers now in the middles of edges) and based on the substitution scheme, applied to each non-straight segment between markers, Here, the top and the bottom choices apply to a concave and a convex vertex,

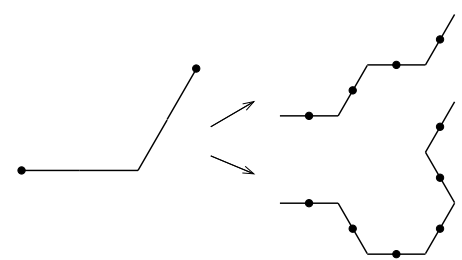

respectively. (A vertex is convex (resp. concave) if one makes a right (resp. left) turn at it while moving counterclockwise on the curve.)

In other words, the crystal oscillates between hexagonal shapes and other familiar mathematical forms with increasingly complex boundary. To illustrate the scientific insight offered by such simple CA rules, Wolfram makes the following intriguing prediction based on his time trace of Hex 1 up to time 30 [Wol3]:

"For example, one expects that during the growth of a particular snowflake there should be alternation between tree-like and faceted shapes, as new branches grow but then collide with each other. And if one looks at real snowflakes, there is every 
indication that this is exactly what happens. And in fact, in general the simple cellular automaton shown above seems remarkably successful at reproducing all sorts of obvious features of snowflake growth."

We intend to address the level of realism of digital snowflakes and other lattice models for ice crystal growth in a sequel to this paper [GG2]. Here our goal is a rigorous study of the complete family of Packard rules. For instance, we will show that each crystal fills the lattice with a characteristic asymptotic density, independent of the finite initial seed. We also identify a subclass of exactly solvable rules for which the density is a computable rational number (e.g., Hex 135 and Hex 134 have densities 5/6 and 21/22, respectively), and a complementary class with inherently more computational complexity of the limit state $A_{\infty}$. Ultimately, our story here has two main morals:

- As CA dynamics go, Packard's digital snowflakes are not very complex. The patterns they trace are not periodic, but nearly so, and in some cases exactly described by a finite recursion. Consequently, they are quite amenable to mathematical methods.

- While computer visualization and empirical calculation are indispensable tools, subtle properties of these dynamics cannot possibly be gleaned from simulations alone. Deductive reasoning plays a fundamental role in the analysis.

Turning to formalities, our basic set-up features solidification CA on the triangular lattice $\mathbb{T}$ (to reflect the arrangement of water molecules in ice crystals). ${ }^{1}$ For notational and computational convenience, we represent $\mathbb{T}$ by $\mathbb{Z}^{2}$, with the neighborhood $\mathcal{N}$ of $(0,0)$ consisting of itself and the six sites $( \pm 1,0),(0, \pm 1)$, and $\pm(1,1)$. The neighborhood of an arbitrary $x$ is then $x+$ $\mathcal{N}$. This representation is handy because integers are much more familiar than Cayley graph representations of $\mathbb{T}$. On the other hand, some symmetries are not so easy to spot. (Figs. 1 and 8 are the only ones in which dynamics on $\mathbb{T}$ are depicted.) The CA simulator MCell [Woj] is ideally suited for empirical investigation of digital snowflakes since its Weighted Life rule set supports this $\mathbb{Z}^{2}$ embedding. Indeed, MCell was a crucial resource during the early stages of our work, and any conscientious reader of this paper will surely need to enlist its aid, or that of some similar program.

We denote by $A_{t} \subset \mathbb{Z}^{2}$ the set of occupied sites at time $t$. Often we call sites in $A_{t} 1$ 's and sites in $A_{t}^{c} 0$ 's. The set $A_{t}$ grows in discrete time $t=0,1,2, \ldots$ That is, $A_{t} \subset A_{t+1}$; such CA are called solidifying. Whether $x \notin A_{t}$ belongs to $A_{t+1}$ depends only on the number of sites it sees in $A_{t}$, that is, on $\left|(x+\mathcal{N}) \cap A_{t}\right|$. Thus, the rule is given by a function $\pi:\{1,2,3,4,5,6\} \rightarrow\{0,1\}$ such that, for $x \notin A_{t}, \pi\left(\left|(x+\mathcal{N}) \cap A_{t}\right|\right)=1$ iff $x \in A_{t+1}$. As above, we specify $\pi$ by listing all $n$ for which $\pi(n)=1$. Our canonical choice of the initial set is $A_{0}=\{0\}$, although we will also study the dynamics started from an arbitrary finite set, and use assorted infinite initial sets as props. Note that, for any solidification dynamics and every $A_{0}$, the final set $A_{\infty}$ exists as a sitewise limit of $A_{t}$, and $A_{\infty}=\cup_{t \geq 0} A_{t}$.

Our basic assumption on $\pi$, reflecting the fact that tips of a growing snow crystal are favored, is that $\pi(1)=1$. We call solidifying CA with this property digital snowflakes. It is not clear what else should be assumed about $\pi$, so we propose to catalog all possible behaviors of such automata.

\footnotetext{
${ }^{1}$ We have chosen to conform to accepted mathematical terminology, although $\mathbb{T}$ is often called the hexagonal lattice in the popular and scientific literature.
} 
There are 32 candidates for digital snowflakes. Of these, 16 are trivial: when $\pi(2)=1$, the appropriate version of the extreme boundary dynamics (see Section 2) is very easy to analyze, and causes the dynamics to grow a full hexagon from a singleton, while from any other finite seed they grow a full hexagon apart from a region at finite distance from the rays defined by the extreme points of the hexagon. (Many real snowflakes also grow as expanding hexagons, but there is no mystery here.) From now on, we only refer to the remaining 16 rules, i.e., to those with $\pi(1)=1$ and $\pi(2)=0$, as digital snowflakes. Next, let us explain our basic approach and summarize our asymptotic density results.
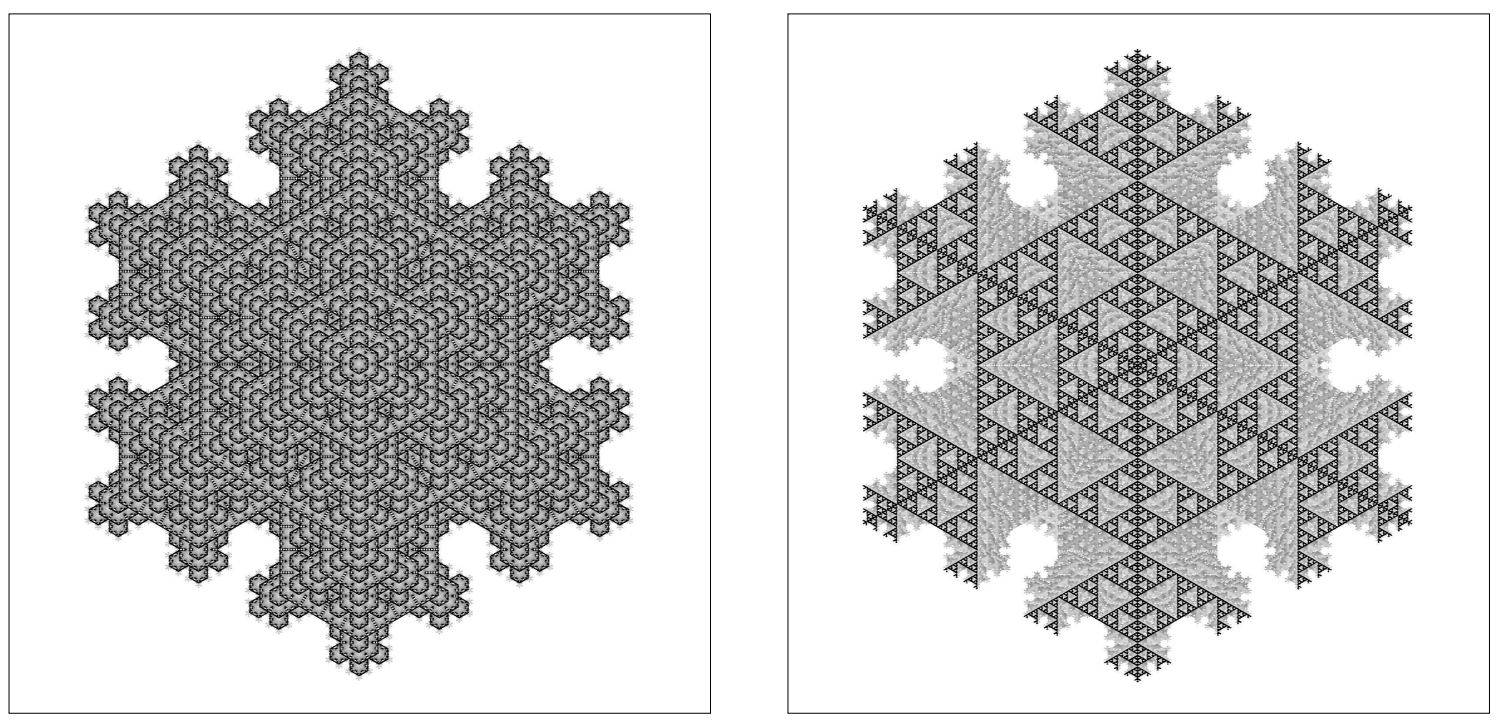

Fig. 1. The occupied set of Hex 134 at time 218, started from $\{0\}$. Let $\tau_{x}=\inf \{t$ : $\left.x \in A_{t}\right\}$ be the time $x$ is occupied and $d$ the distance on the triangular lattice. The two frames depict periodically shaded contours of constant $\tau_{x}$ (left) and constant $\sigma_{x}=\tau_{x}-d(x, 0)$ (right, where only the 0 contour $\left\{x: \sigma_{x}=0\right\}$ is black).

The right frame of Fig. 1 highlights key structural features that underlie our analysis. Note first that any digital snowflake advances at speed 1 along the axes of the lattice, since the closest unoccupied site in these directions always has only one occupied neighbor. Within each of six wedges formed by the axes, the black cells are those that solidify at the edge of the light cone, i.e., at the maximum speed of propagation allowed by a nearest neighbor rule. This process induces symmetric copies of the space-time pattern of a one-dimensional CA. Because $\pi(1)=1$ and $\pi(2)=0$, this is the additive xor rule, arguably the most familiar of all cellular automata. Consequently the black cells form discrete versions of a famous fractal known as the Sierpinski triangle. Of course digital snowflakes continue to solidify after the edge of the light cone passes, as seen in the gray portions of the right frame of Fig. 1. But the Sierpinski lattice effectively divides the crystal into independent finite regions with all 1 boundary conditions, within which subsequent dynamics evolve.

To summarize, Packard's snowflakes enjoy three important properties (to be precisely described in Section 2):

- Starting from a single occupied cell, the light cone CA forms an impenetrable web of occupied sites that divides further solidification into independent finite domains with simple 
boundary conditions;

- Boundary effects within each domain are controlled;

- The light cone CA is additive, so the web from a general finite seed is representable as a superposition of webs from each of its individual cells.

The first two properties ensure a recursive representation of the dynamics, while the last is crucial for the asymptotic density's independence of $A_{0}$.

The delicacy of our results is conveyed effectively by comparison to analogous solidification on $\mathbb{Z}^{2}$ with range 1 Box neighborhood consisting of a central cell and its 8 nearest neighbors: $(0,0),(0, \pm 1),( \pm 1,0),( \pm 1, \pm 1)$. There are 128 such rules with $\pi(1)=1$; see [Gri] for a brief introduction and colorful graphics of Box 1, 157, 1357, and 136 crystals. Although snowflakelike recursive carpets emerge in a great many cases, any and all of the three properties above may fail. For instance, we will see in Section 6 that the density of Box 1, provided it exists at all, can depend on the initial seed. Also, for the "odd" rule, Box 1357, the light cone web "leaks" and growth is apparently chaotic. Although there are many fascinating problems connected with the Box neighborhood, and exact computations are feasible in some cases, there is no hope of a complete analysis as in the present Hex setting.

Fix a set $S \subset \mathbb{Z}^{2}$. Let $\mu_{\epsilon}$ be $\epsilon^{2}$ times the counting measure on $\epsilon \cdot S$. We say that $S$ has asymptotic density $\rho$ if $\mu_{\epsilon}$ converges to $\rho \cdot \lambda$ as $\epsilon \rightarrow 0$. Here $\lambda$ is Lebesgue measure on $\mathbb{R}^{2}$ and the convergence holds in the usual sense:

$$
\int f d \mu_{\epsilon} \rightarrow \rho \cdot \int f d \lambda
$$

for any $f \in \mathcal{C}_{c}\left(\mathbb{R}^{2}\right)$.

Theorem 1. To each of the 16 digital snowflakes there corresponds a $\rho \in(0,1]$, the asymptotic density of $A_{\infty}$, which is independent of the finite seed $A_{0}$.

We will index the densities by our notation for the respective rules, and give more information on their values in the next theorem.

Theorem 2. The densities are exactly computable in 8 cases:

$$
\begin{aligned}
& \rho_{13}=\rho_{135}=5 / 6 \approx 0.8333, \\
& \rho_{134}=\rho_{1345}=21 / 22 \approx 0.9545, \\
& \rho_{136}=\rho_{1356}=\rho_{1346}=\rho_{13456}=1 .
\end{aligned}
$$

In six other cases, one can estimate, within \pm 0.0008 ,

$$
\begin{aligned}
& \rho_{1} \approx 0.6353, \\
& \rho_{14}, \rho_{145} \approx 0.9689, \\
& \rho_{15} \approx 0.8026, \\
& \rho_{16} \approx 0.7396, \\
& \rho_{156} \approx 0.9378 .
\end{aligned}
$$

Finally,

$$
\rho_{146} \in(0.995,1), \rho_{1456} \in(0.9999994,1) .
$$


Perhaps surprisingly, $\rho_{14}>\rho_{134}$, testimony to the fundamentally nonmonotone nature of these rules.

We refer to the first 8 rules in Theorem 2 as exactly solvable. In Section 9, we will develop a rigorous foundation for this terminology.

It is tempting to conjecture that $\rho_{14}=\rho_{145}$ and $\rho_{146}=\rho_{1456}$ since the two dynamics of each pair are identical starting from $A_{0}=\{0\}$ on finite arrays up to $500 \times 500$ in size. This question remains open, but one should resist such empirical conclusions. For instance, as we shall see later, observing Hex 1456 from $A_{0}=\{0\}$ on even the world's most extensive graphics array, with millions of sites on a side, one would still be led to the conclusion that $A_{\infty}=\mathbb{Z}^{2}$. In fact, the four dynamics are all distinct eventually and presumably have different densities less than one.

In contrast to density results, which are macroscopic in nature, our next result addresses the most basic microscopic properties of final configurations. Call a set $S \subset \mathbb{Z}^{2}$ thick if

$$
\sup \left\{d(x, S): x \in \mathbb{Z}^{2}\right\}<\infty .
$$

Here, $d$ is distance in any chosen norm, say $\|\cdot\|_{\infty}$. For snowflakes with density between 0 and 1 , thickness of $A_{\infty}$ and $A_{\infty}^{c}$ is one rough notion of an almost periodic final state. In the following theorem, $A_{0}$ is assumed to be an arbitrary finite set.

Theorem 3. The 8 exactly solvable rules have the following properties:

(1) The final set $A_{\infty}$ is always thick.

(2) Hex 13456 always has $A_{\infty}=\mathbb{Z}^{2}$. For the other rules with density 1 , there exist initial conditions for which $A_{\infty}$ contains infinitely many 0's.

(3) $A_{\infty}^{c}$ is always thick for rules with density $<1$, and never thick for those with density 1.

For the 8 rules that are not exactly solvable, $A_{\infty}$ is never thick, and Hex 1 always has thick $A_{\infty}^{c}$.

It is an intriguing open question whether $A_{\infty}^{c}$ is thick for the 7 rules not covered by Theorem 3. We suspect it is for all of them, but have no argument.

The rest of the paper is organized as follows. Preliminaries in Section 2 describe precisely various structural properties of the additive web that decomposes a digital snowflake into independent regions of finite size. Slight variations in how these regions solidify are identified case by case. Sections 3-4 then detail the first 8 density calculations of Theorem 2 by deriving and solving recursions for $\left|A_{2^{n}-1}\right|$. Section 3 handles exactly solvable cases - first the simplest rules: $13,135,136$, and 1356 , and then those obeying slightly more complicated dynamics: $134,1345,1346$, and 13456. For all these snowflakes the limit of $\left|A_{2^{n}-1}\right| /\left(3 \cdot 4^{n}\right)$ is evaluated explicitly. Next, Sections 4-5 develop and analyze corresponding recursions for Hex 1 and the other 7 rules that are not exactly solvable. Now, due to certain messy interactions, existence of the normalized limit of occupied cells is established by a novel application of the renewal theorem, but this density is implicit and can only be approximated numerically. Section 4 handles six densities that we are able to estimate within 0.0008. The cases 146 and 1456 in Section 5 require a different rescaling argument for the upper bound since their densities are extremely close to, but less than, 1 .

In Section 6 we complete the proof of Theorem 1 by showing that $A_{\infty}$ has an asymptotic density $\rho$ starting from $\{0\}$ in the formal sense of (1.1), that $\rho$ agrees with the corresponding 
value obtained in Sections 3-6, and that the same asymptotic density occurs when the initial seed is an arbitrary finite set $A_{0}$. (A technicality for rules with $\pi(3)=0$ is also handled at the end of this section.) Then, Section 7 introduces two distinct, rule-dependent macroscopic dynamics $\mathcal{S}_{a}$ for the limit of $2^{-n} A_{t_{n}}$, where $t_{n}=\left\lfloor a \cdot 2^{n}\right\rfloor$. Theorems 4 and 5 thereby extend to general $a$ the already mentioned substitution scheme limit for Hex 1 and Hex 135 in the case $a=1 / 3$. A more sophisticated approach is required to handle both holes formed by colliding branches of the snowflake and the case of irrational $a$. Examples are given to illustrate the exotic dependence on $a$ of the Hausdorff dimension of the boundary of $\mathcal{S}_{a}$. Finally, Sections 8 and 9 address thickness (Theorem 3) and exact solvability, respectively. Theorems 1-3 yield a natural and precise division of digital snowflakes into two complexity classes (Section 9). This distinction, based on the notion of automaticity of the final set, is potentially widely applicable in the computational theory of cellular automata.

\section{Extreme boundary dynamics}

Our basic tool is the additive dynamics $T_{n}$, also referred to as xor, addition mod 2, or rule 90 (see, e.g., [Wil]). Among several equivalent definitions, we choose the following. We declare the neighborhood of 0 to consist of $(-1,0)$ and $(-1,-1)$, and the additive rule to be exactly one solidification dynamics with this two-point neighborhood, i.e., according to this rule a site changes its state to 1 iff exactly one of $x+(-1,0)$ and $x+(-1,-1)$ is in state 1 . The initial set $T_{0}$ will always be a subset of the $y$-axis, our default choice being the singleton $\{0\}$. If we want to emphasize that $T_{0}=A$, we use the notation $T_{n}^{A}$. Many properties of this rule are well known and easy to check. Nevertheless, we will explain them briefly as they are used.

Observe first that, with the canonical choice $T_{0}=\{0\}$,

$$
\{(x, 0): 0 \leq x \leq n\} \cup\{(x, x): 0 \leq x \leq n\} \subset T_{n} \subset\{(x, y): 0 \leq y \leq x \leq n\} .
$$

One can also prove by induction that

$$
\begin{aligned}
& T_{2^{n}-1} \cap\left\{(x, y): x=2^{n}-1\right\}=\left\{\left(2^{n}-1, y\right): 0 \leq y \leq 2^{n}-1\right\}, \\
& T_{2^{n}} \cap\left\{(x, y): x=2^{n}\right\}=\left\{\left(2^{n}, 0\right),\left(2^{n}, 2^{n}\right)\right\},
\end{aligned}
$$

since the two "buds" at time $2^{n}$ create two versions of the dynamics that do not interact through time $2^{n+1}-1$. The name of the dynamics stems from the fact that it preserves exclusive union: $T_{n}^{A \operatorname{xor} B}=T_{n}^{A}$ xor $T_{n}^{B}$. This is immediate for $n=1$, and then again follows by induction.

It is helpful to consult Figs. 2-4 while reading the remainder of this section. The darker sites in those figures form initial conditions (the reasons for which will be explained later). For growth from a single seed at the origin, the lowest row (resp. 2 rows) in the top three frames of Figs. 2 and 3 (resp. Fig. 4) should be deleted, and then the origin placed at the leftmost lowest site. Also, time should be diminished by 1 in Figs. 2 and 3.

The relevance of additive dynamics to digital snowflakes becomes apparent when we note that if $A_{0}$ does not include any site to the right of the $y$-axis,

$$
A_{n} \cap\{(x, y): x=n\}=T_{n}^{A_{0} \cap y \text {-axis }} \cap\{(x, y): x=n\}
$$

in any of our 16 dynamics. For a general solidification CA, the light cone of a set $L_{0}$ is the set $L_{n}=L_{0}+\mathcal{N}+\mathcal{N}+\cdots+\mathcal{N}$, where $\mathcal{N}$ is repeated $n$ times, i.e., the set of points than can 
possibly be influenced by $L_{0}$ at time $n$. In particular, if $L_{0}=A_{0}$, which we assume from now on, $A_{n} \subset L_{n}$. The extreme boundary comprises sites $y \in L_{n}$ with $(x+\mathcal{N}) \cap L_{n}^{c} \neq \emptyset$. Then (2.4) means that our rules perform six copies of the additive dynamics (appropriately mapped) at their extreme boundaries, and create an "additive web" consisting of black sites $x$ with $\sigma_{x}=0$ in the right frame of Fig. 1. We will call this the extreme boundary dynamics, and the sites so created primary boundaries. Also note that properties (2.1-2.2) dictate impenetrable boundaries for our rules. For example, if $A_{0}=\{0\}$, then after time $2^{n}-1$ the dynamics create a triangle of 1's given by (2.1) and (2.2) and after that time the dynamics inside and outside the triangle are independent. Even (2.1) itself, as it extends all the way to the extreme boundary, separates the dynamics into six independent wedges. For many purposes, then, it will suffice to look at one of these wedges, typically the one in the first quadrant and below the line $y=x$.

Digital snowflakes do more than add sites at their extreme boundaries. At time $2^{n}$, for example, the two buds in (2.3) do not merely spread into two new triangles outlined by the additive dynamics; they also grow into the empty triangle between them. To be more precise, in the 8 rules with $\pi(3)=1$, there is a pair of occupied sites $\left(2^{n}, 1\right),\left(2^{n}, 2^{n}-1\right) \in A_{2^{n}+1}$; in the remaining 8 cases, $\left(2^{n}+1,2\right),\left(2^{n}+1,2^{n}-1\right) \in A_{2^{n}+2}$. In fact, it is more convenient to interpret the initial buds as being at sites below and above these two - they are marked $\bullet$ in the top left configurations of Figs. 2 and 4. As we explain below, these two buds generate their own secondary extreme boundary dynamics (spreading into the two smaller triangles outlined in the figures) until they collide.

Assume first that $\pi(3)=1$. Then the two buds generate exactly (appropriately rotated and deformed) additive dynamics at their extreme boundaries. This is because one of the two endpoints (sites generating the leftmost set in (2.1)) sees 3 occupied sites, two of which are contributed by the boundary conditions (occupied sites in primary boundaries), while the other endpoint is shared by the neighboring additive dynamics. Therefore, the said two extra buds generate two copies of additive extreme boundary dynamics (in the smaller triangles), which at the time $2^{n}+2^{n-1}-1$ generate an occupied secondary row and diagonal of length $2^{n-1}$, separated by a 0 at $\left(2^{n}, 2^{n-1}\right)$. This 0 , let us call it the four-site, sees 4 occupied sites.

If $\pi(4)=0$, the four-site (marked by $\circ$ in the top middle of Fig. 2) will not get occupied immediately, and the vertex of the wedge between the row and the diagonal of 1's is "dead." The two secondary boundaries, together with the primary ones $\left\{\left(x, 2^{n}\right),\left(x, x-2^{n}\right): 2^{n}+2^{n-1} \leq\right.$ $\left.x \leq 2^{n+1}-1\right\}$ form a "hole," which is invaded by dynamics that emanate from two one-buds at $\left(2^{n}+2^{n-1}, 2^{n-1}\right)$ and $\left(2^{n}+2^{n-1}, 2^{n}\right)$, created at time $2^{n}+2^{n-1}$ (each marked by $\bullet$ in the top middle of Fig. 2). This hole is in turn divided into two smaller holes (by secondary 1's) at time $2^{n}+2^{n-1}+2^{n-2}-1$, etc. This hole-filling mechanism is illustrated in the bottom of Fig. 2 , where the two descendant holes are outlined in the first two frames. It is important to note that the parallelogram hole in the top row of this figure is equivalent, modulo boundary corrections, to the small square hole with darker shaded 1 boundary conditions. (Match the marked first two occupied sites with the same marks in the parallelogram hole.) This consequence of symmetries of $\mathbb{T}$ will be exploited throughout. Armed with these observations, we will begin our study of the Hex 13 rule in the next section.

We emphasize that some of the sites which we use as initial conditions are not present initially; the hole "frames" are typical examples. Indeed, some of these initial sites may never be created, as in the Hex 1 case. However, they are very convenient for definitions and for symmetry considerations. In each case, it is straightfoward to verify that the dynamics behaves as if these sites were present initially. 
On the other hand, if $\pi(3)=\pi(4)=1$, then the four-site (now marked $\bullet$ in Fig. 3) becomes occupied at time $2^{n}+2^{n-1}$ and the resulting "live" vertex gives rise to another secondary dynamics in the hole. Three secondary boundary dynamics originating at the four-site and the two one-buds all collide at time $2^{n}+2^{n-1}+2^{n-2}-1$ to create a triangularly shaped hole (as in the middle top of Fig. 3). The remaining two secondary boundary dynamics collide at the same time and create a smaller quadrilateral hole. This one, however, is of a different type than the original, as only two of its vertices are live. This mechanism is iterated, as illustrated by a larger hole example in Fig. 3. Our analysis of Hex 134 dynamics will therefore require three types of hole dynamics, generated by different initial conditions.

The situation is again different when $\pi(3)=0$. Now the two secondary buds are a little off center (by one site, to be precise). They still generate additive boundary dynamics, but the final interaction inside the resulting hole generates two holes of different sizes, and each successive generation of holes has one of a smaller size. We give a more precise description for the Hex 1 rule; others are similar. In this case, all points $\left(2^{n}, y\right), 1 \leq y \leq 2^{n}-1$, see at least 2 occupied sites to their left at time $2^{n}-1$ (hence thereafter) and thus will never get occupied. The two secondary buds collide at time $2^{n}+2^{n-1}$, but the secondary row and diagonal (created at time $\left.2^{n}+2^{n-1}\right)$ are now not separated. Nevertheless, the 0 at $\left(2^{n}+2,2^{n-1}+1\right)$ (marked $\circ$ in Fig. 4) sees 4 occupied points, so it never gets occupied and the resulting vertex of the wedge is dead ${ }^{2}$. The two secondary boundaries, together with primary ones, create a hole. This hole starts being filled by buds that appear at time $2^{n}+2^{n-1}+2$ at $\left(2^{n}+2^{n-1}+1,2^{n-1}+1\right)$ and $\left(2^{n}+2^{n-1}+1,2^{n}\right)$. The fact that these buds are off center has two consequences. The first is minor and technical: it is necessary to start the analysis with a basic wedge and holes that incorporate the buds in their initial conditions. The second fact is crucial for the analysis of these rules. Namely, the two holes that result when the additive dynamics from two secondary buds collide are of unequal size, their sizes differing by exactly 2 . This in turn creates holes of a larger and larger variety of sizes (as shown in the two bottom frames of Fig. 4, where the second generation descendant holes are also outlined, since they evolve slightly out of phase and are thus difficult to identify). That the interaction in smaller holes still creates impenetrable boundaries and dead wedge vertices is guaranteed by the following lemma.

Lemma 2.1. Assume that the initial configuration consists of two points, $A_{0}=\left\{(0,0),\left(2^{n}-\right.\right.$ $\left.\left.k, 2^{n}-k\right), 0<k \leq 2^{n-1}\right\}$. For any digital snowflake,

$$
\left\{\left(y, 2^{n}-1\right): 0 \leq y \leq 2^{n-1}-k+1\right\} \subset A_{2^{n}-1} .
$$

Proof. This is a simple consequence of the speed of light. Namely, consider the light cone of the point $\left(2^{n}-k, 2^{n}-k\right)$ at time $t$. Outside this light cone, the dynamics started from $\{(0,0)\}$ and the one started from $A_{0}$ agree through time $t$. Apply this observation at time $t=2^{n}-1$.

\section{Densities for exactly solvable rules}

We begin with the Hex 13 rule, as it represents the simpler of two exactly solvable cases with nontrivial density.

Assume first that the initial occupied set is the origin, $A_{0}=\{0\}$, and define

$$
\rho_{13}=\lim _{n \rightarrow \infty} \frac{\left|A_{2^{n}-1}\right|}{3 \cdot 4^{n}}
$$

\footnotetext{
${ }^{2}$ In rules with $\pi(4)=1$, such as $\operatorname{Hex} 14$, this 0 becomes a 1 , but then growth from the vertex stops.
} 
We will see shortly that the limit exists, and that

$$
\rho_{13}=\lim _{n \rightarrow \infty} \frac{b_{n}}{4^{n}},
$$

where $b_{n}$ are defined by certain wedge dynamics. Namely, run the dynamics $A_{n}$ inside the first quadrant with 1 boundary condition on the axes. Let

$$
B_{n}=\left\{(x, y): 1 \leq x \leq 2^{n}, 1 \leq y \leq 2^{n}\right\} .
$$

Then $b_{n}$ is the size of the final occupied set inside $B_{n}$,

$$
b_{n}=\left|A_{\infty} \cap B_{n}\right|=\left|A_{2^{n}} \cap B_{n}\right| .
$$

The second equality, i.e., the fact that these dynamics do not occupy any site on $B_{n}$ past time $2^{n}$, can be proved by induction along the lines of arguments given below.
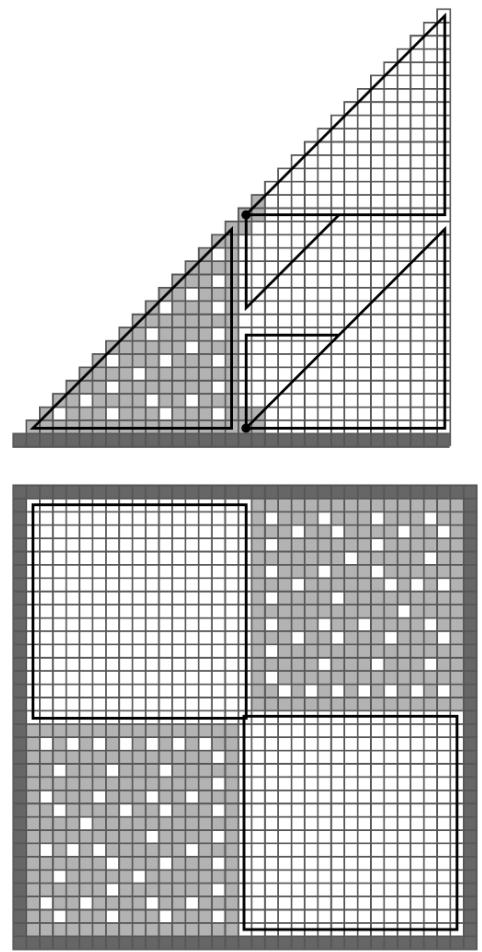
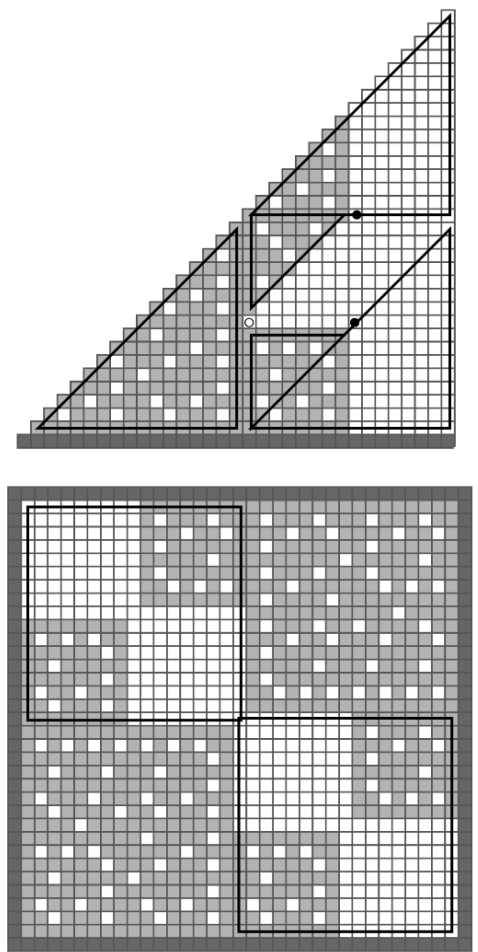
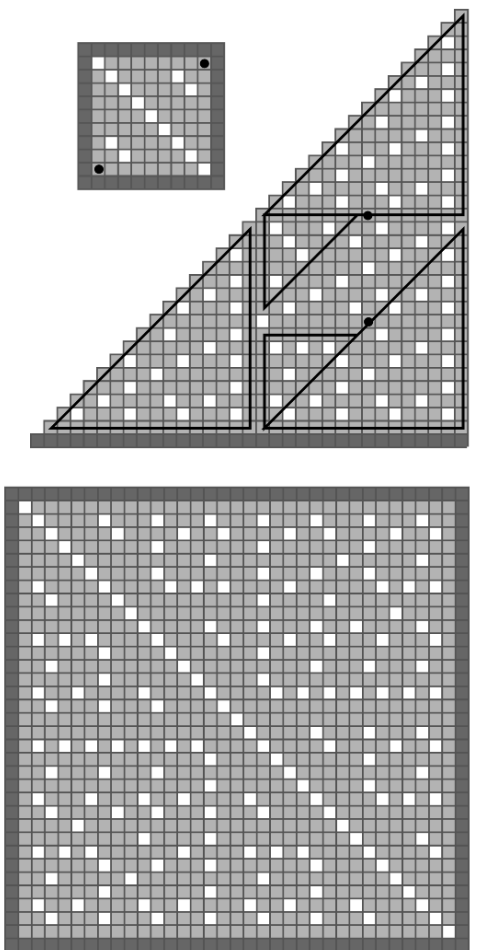

Fig. 2. Hex 13: Basic wedge dynamics (top) at times 18, 24, and 32; final configuration of a hole of size 10; size 34 hole dynamics at times 16,24 , and 32 .

Smaller wedge numbers are obtained with the same initial condition as for $b_{n}$ but counted only inside

$$
W_{n}=\left\{(x, y): 1 \leq x \leq 2^{n}, 1 \leq y \leq x\right\}
$$

and defined by

$$
w_{n}=\left|A_{\infty} \cap W_{n}\right| .
$$

The dynamics inside this wedge (with given initial conditions) will be called the basic wedge dynamics. See the top row of Fig. 2. Note that $b_{n}=2 w_{n}-2^{n}$. 
By direct enumeration, $b_{0}=1, b_{1}=4, b_{2}=14, b_{3}=54$, etc. Moreover, as $A_{2^{n}-1}$ started from $A_{0}=\{0\}$ contains, modulo boundary corrections, six copies of the dynamics on $W_{n}$,

$$
\left|A_{2^{n}-1}\right|=3 b_{n}-3 \cdot 2^{n}+1 .
$$

Next, consider the dynamics on the $\{0, \ldots, L\} \times\{0, \ldots, L\}$ box with initial occupied sites consisting of the frame $\{(x, y)$ : either $x \in\{0, L\}$ or $y \in\{0, L\}\}$. We call $L$ the size of such a hole. Holes of sizes 10 and 34 are featured in Fig. 2. Let $H(L)$ be the final occupation count minus the frame,

$$
H(L)=\left|A_{\infty} \cap\{(x, y): 0<x<L, 0<y<L\}\right| .
$$

We define

$$
h_{n}=H\left(2^{n}+2\right) .
$$

Thus $h_{0}=2, h_{1}=6, h_{2}=20$, etc. Actually it is slightly more convenient to use $h_{n}^{\prime}=h_{n}-2^{n+1}$. By observing the wedge dynamics from time $2^{n}$ to time $2^{n+1}$, we obtain our first basic recursion:

$$
w_{n+1}=3 w_{n}+2\left(w_{n-1}-2^{n-1}\right)+h_{n-1}^{\prime} .
$$

This recursion is illustrated by the top row of Fig. 2, where the 5 smaller wedges are outlined. Here one thinks of the sites in $\mathbb{Z}^{2}$ as centers of the squares, and the outlined regions include their boundaries. The sites outside the outlined regions correspond to the interior of the pictured size 10 hole, from which the bottom row and rightmost column are removed. Therefore

$$
b_{n+1}=3 b_{n}+2 b_{n-1}+2 h_{n-1}^{\prime} .
$$

A second basic recursion is obtained by observing the hole dynamics until time $2^{n-1}$ :

$$
h_{n}=2 b_{n-1}+2 h_{n-1} \text {. }
$$

This is illustrated in the bottom row of Fig. 2, where the interiors of the first descendant holes are outlined. Equivalently,

$$
h_{n}^{\prime}=2 b_{n-1}+2 h_{n-1}^{\prime} .
$$

Express $h_{n}^{\prime}$ in terms of $b$ 's using (3.2), and then plug into (3.3) to obtain the second order equation $b_{n+2}=5 b_{n+1}-4 b_{n}$, valid for $n \geq 1$. Hence $b_{n}=\alpha \cdot 4^{n}+\beta$. Computing the constants $\alpha, \beta$ from $b_{1}$ and $b_{2}$, we get

$$
b_{n}=\frac{5}{6} \cdot 4^{n}+\frac{2}{3} .
$$

Thus, starting from $A_{0}=\{0\}$, by (3.1),

$$
\left|A_{2^{n}-1}\right|=\frac{5}{2} \cdot 4^{n}-3 \cdot 2^{n}+3,
$$

and $\rho_{13}=\frac{5}{6}$.

Hex 135, Hex 136, and Hex 1356 all satisfy precisely the same recursions (3.2)-(3.3). Also, it is easy to check that for small holes and wedges Hex 13 and Hex 135 evolve identically. Thus, it follows by induction that Hex 13 and Hex 135 crystals agree exactly for all $t$ when $A_{0}=\{0\}$. In particular, $\rho_{135}=\rho_{13}=\frac{5}{6}$. Since Hex 136 and Hex 1356 both fill small holes and wedges, it also easily follows that these two rules solidify completely from a singleton. 

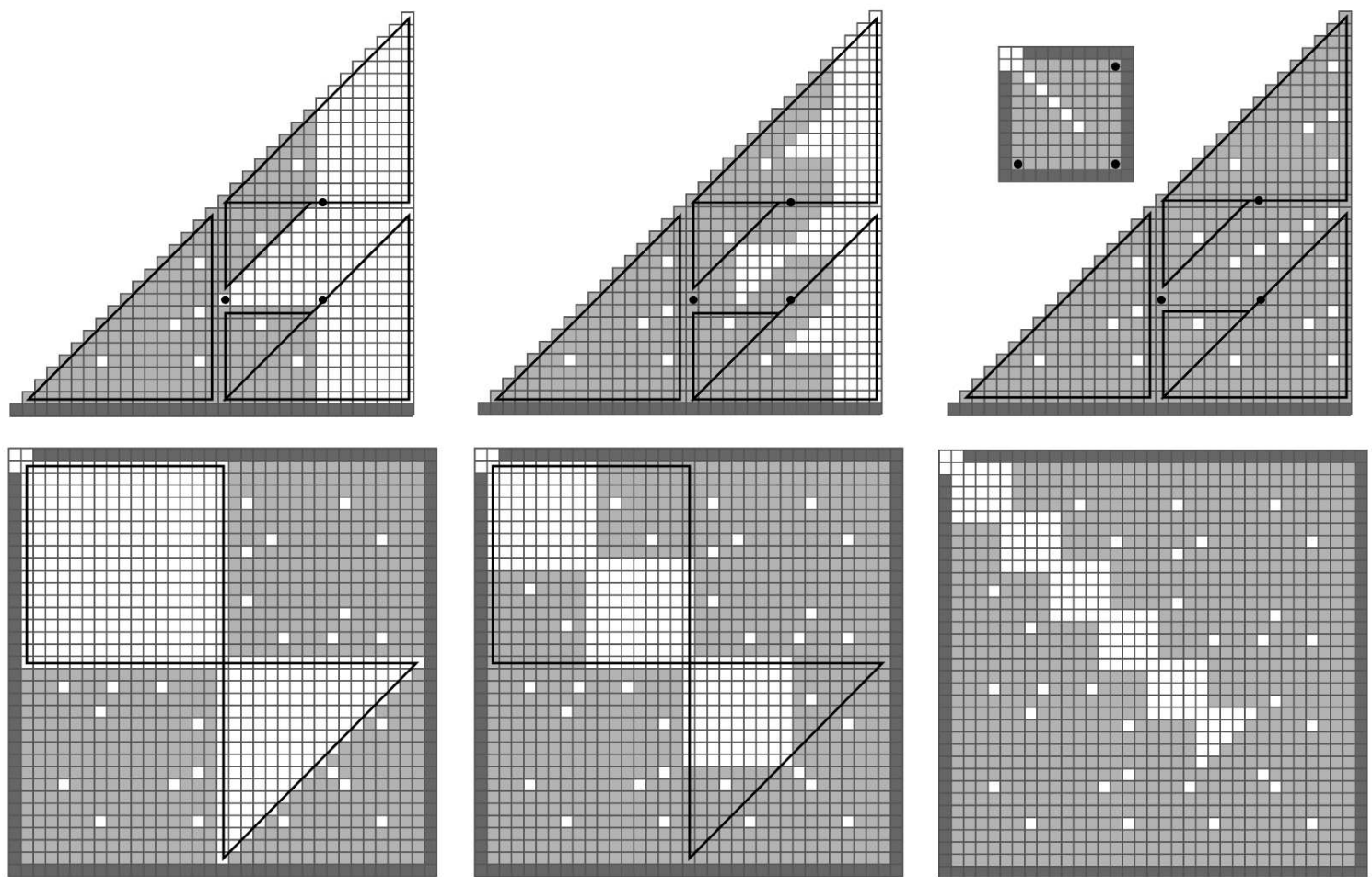

Fig. 3. Hex 134: Basic wedge dynamics (top) at times 24, 28, and 32; final configuration of a hole of size 10; size 34 hole dynamics at times 16, 24, and 28.

We turn next to Hex 134, another exactly solvable rule with density less than 1 . The domain counts $b_{n}$ and $w_{n}$ are defined in exactly the same manner as before, giving $b_{0}=1, b_{1}=4, b_{2}=16$, $b_{3}=62, b_{4}=246$, etc.

As mentioned in Section 2, the hole interactions (see Fig. 3) are more complicated now. There are three different types of hole dynamics, all run in the $\{0, \ldots, L\} \times\{0, \ldots, L\}$ box. We define the frame $F=\{(x, y): x \in\{0, L\}$ or $y \in\{0, L\}\}$. All occupation numbers are counted strictly inside this frame, and in addition exclude any other initially occupied sites. We will always use $L=2^{n}+2$ and count occupation numbers at time $2^{n}$ (which in every case differ by 1 from the final occupation numbers).

The principal hole dynamics use initial occupied sites consisting of the "frame minus 3 corner sites": $F \backslash\{(0, L-1),(0, L),(1, L)\}$, and defines the occupation numbers $h_{n}$. Then $h_{0}=3, h_{1}=7$, $h_{2}=22$, etc. Again, we set $h_{n}^{\prime}=h_{n}-2^{n+1}$.

The secondary hole dynamics start with two opposite corners missing: $F \backslash\{(0, L-1)$, $(0, L),(1, L),(L-1,0),(L, 0),(L, 1)\}$. Call the resulting occupation numbers $s_{n}$. Then $s_{0}=2$, $s_{1}=6, s_{2}=20$, etc.

The third and last hole dynamics start with a triangular shaped hole, i.e., the initially occupied set $(F \backslash\{(0, L-1),(0, L),(1, L)\}) \cup\{(x, y): y<x\}$, and define $t_{n}$. Then $t_{0}=2, t_{1}=5$, $t_{2}=13$, etc. 
Our system of recursions is given by (3.2), again illustrated in the top frames of Fig. 3, and

$$
\begin{aligned}
& h_{n}=2 b_{n-1}+w_{n-1}+t_{n-1}+s_{n-1} \\
& s_{n}=2 b_{n-1}+2 s_{n-1} \\
& t_{n}=2 w_{n-1}+h_{n-1} .
\end{aligned}
$$

To understand these new equations, consult the bottom row of Fig. 3. The first successor holes (outlined) are a triangular one and a square one of secondary type. The former creates a hole of the principal type, while the latter creates two holes of the secondary type, as seen in the bottom middle frame. Therefore, we have

$$
\begin{aligned}
& h_{n}^{\prime}=\frac{5}{2} b_{n-1}+t_{n-1}+s_{n-1}-\frac{7}{4} 2^{n} \\
& s_{n}=2 b_{n-1}+2 s_{n-1} \\
& t_{n}=b_{n-1}+h_{n-1}^{\prime}+\frac{3}{2} 2^{n} .
\end{aligned}
$$

At this point the problem could be solved by matrix manipulation, but it is easier to eliminate $s_{n}$ and $t_{n}$ using the first and third equations of (3.4), then eliminate $h_{n}^{\prime}$ using (3.2). This yields the equation $b_{n+3}-5 b_{n-2}+3 b_{n-1}+4 b_{n}=0$. It follows that $b_{n}$ is a linear combination of $4^{n}, \phi^{n}$, and $\left(-\phi^{-1}\right)^{n}$, where $\phi=(1+\sqrt{5}) / 2$ is the golden ratio. Computing constants, the final result is, for $n \geq 1$,

$$
b_{n}=\frac{21}{22} \cdot 4^{n}+\frac{15-\sqrt{5}}{55} \cdot\left(\frac{1+\sqrt{5}}{2}\right)^{n}+\frac{15+\sqrt{5}}{55} \cdot\left(\frac{1-\sqrt{5}}{2}\right)^{n} .
$$

Again (3.1) holds, so $\rho_{134}=\frac{21}{22}$.

Once more, it is easy to prove that from $A_{0}=\{0\}$, Hex 1345 generates exactly the same $A_{\infty}$ as Hex 134, while Hex 1346 and Hex 13456 solidify completely.

\section{Density of $\operatorname{Hex} 1$ and its cousins}

In this section we will analyze, in this order, Hex 1, 14, 145, 15, 16, and 156. We still use the definition,

$$
\rho=\lim _{n \rightarrow \infty} \frac{\left|A_{2^{n}-1}\right|}{3 \cdot 4^{n}},
$$

where $A_{0}=\{0\}$. However, for these rules we cannot give the exact value of the density; instead we will demonstrate that the limit exists. It is, for now, convenient to redefine

$$
\rho=\lim _{n \rightarrow \infty} \frac{b_{n}}{4^{n}} ;
$$

we prove that (4.1) also holds at the end of Section 6 . The numbers $b_{n}$ are defined by appropriate wedge dynamics, defined slightly differently than before. Namely, these dynamics run inside the first quadrant with 1 boundary condition on the axes, and with the initial occupied set $A_{0}$ consisting of the single point (apart from the axes) $(1,2)$. Now set

$$
B_{n}=\left\{(x, y): 1 \leq x \leq 2^{n}, 1 \leq y \leq 2^{n}+2\right\} .
$$

and let $b_{n}$ be the size of the final occupied set inside $B_{n}$, i.e.

$$
b_{n}=\left|A_{\infty} \cap B_{n}\right| .
$$


We also make use of the smaller wedge

$$
W_{n}=\left\{(x, y): 1 \leq x \leq 2^{n}, 2 \leq y \leq x+1\right\},
$$

and define

$$
w_{n}=\left|A_{\infty} \cap W_{n}\right| .
$$

Note that $b_{n}=2 w_{n}-1-\left(2^{n}-1\right)=2 w_{n}-2^{n}$,

Appropriate hole dynamics on the $\{0, \ldots, L\} \times\{0, \ldots, L\}$ box have initial occupied sites consisting of the frame $\{(x, y)$ : either $x \in\{0, L\}$ or $y \in\{0, L\}\}$ together with $(1,2)$ and $(L-2, L-1)$. As before,

$$
H(L)=\left|A_{\infty} \cap\{(x, y): 0<x<L, 0<y<L\}\right|,
$$

but now we need a much larger variety of hole counts:

$$
h_{n}^{k}=H\left(2^{n}-2 k\right), \quad k=-1,0,1, \ldots
$$

Here we view $k$ as fixed and $n$ large enough that this makes sense. We also abbreviate $h_{n}=h_{n}^{-1}$.

Two basic recursions will be derived in a manner similar to the Hex 13 analysis. The only difference between the six rules is the correction term in (4.2) below. Equation (4.3) is the same in all cases.

We now proceed with Hex 1.
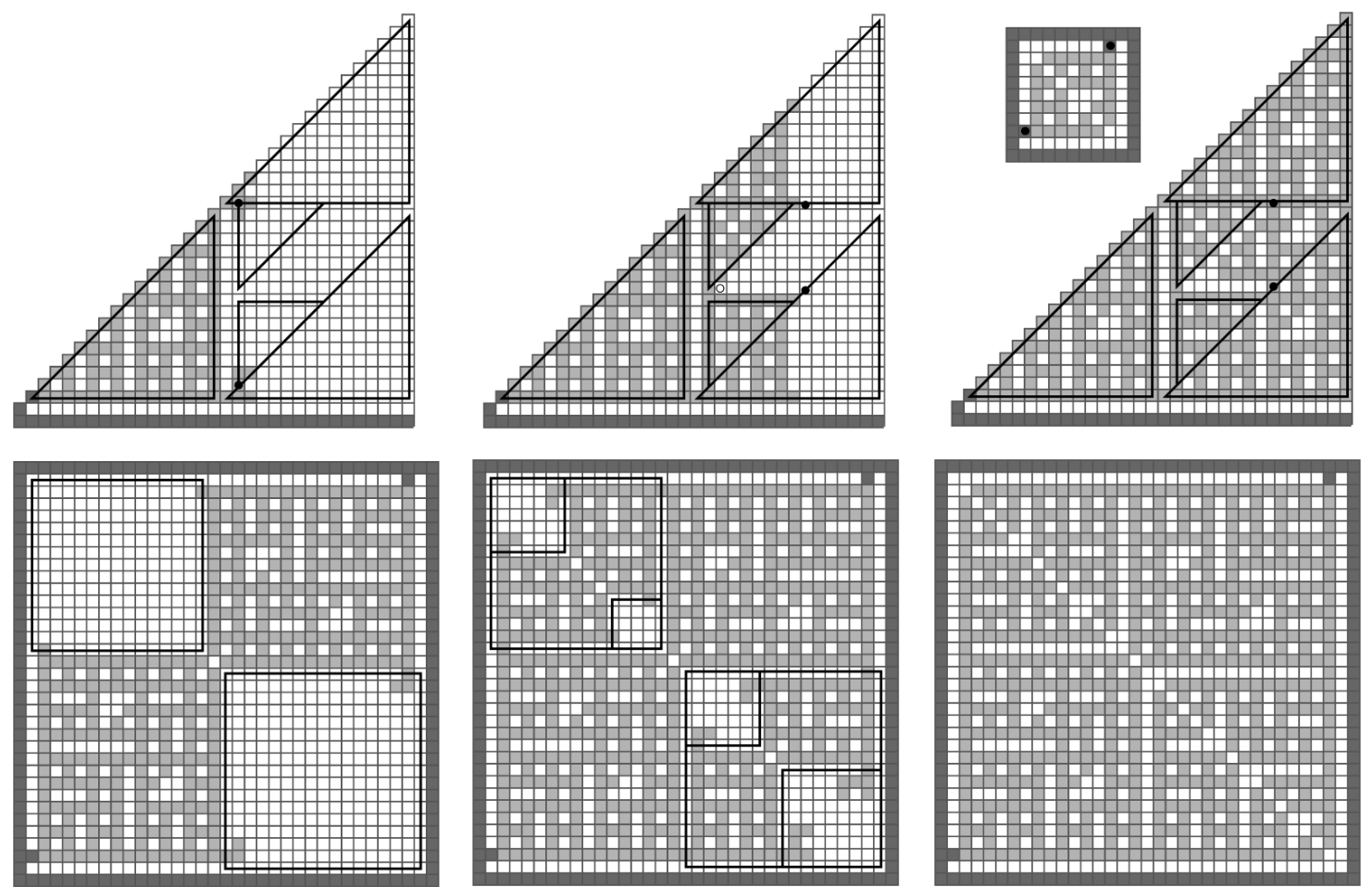

Fig. 4. Hex 1: Basic wedge dynamics (top) at times 18, 24, and 32; final configuration of hole of size 10; size 34 hole dynamics at times 17, 26, and 32 . 
The first recursion is obtained by observing $W_{n}$ from time $2^{n}$ to $2^{n+1}$ :

$$
\begin{aligned}
w_{n+1} & =3 w_{n}+2\left(w_{n-1}-2^{n-1}\right)+h_{n-1}-2\left(2^{n-1}-1\right) \\
& =3 w_{n}+2 w_{n-1}+h_{n-1}-2^{n+1}+2 .
\end{aligned}
$$

Note (as in the top left frame of Fig. 4) that two rows and columns are now removed from the size 10 hole to match the sites not covered by the five wedges. Therefore

$$
b_{n+1}=3 b_{n}+2 b_{n-1}+2 h_{n-1}-2^{n+1}+4 .
$$

For example, $b_{0}=1, b_{1}=4, b_{2}=14, b_{3}=50, b_{4}=182, \ldots ;$ and $h_{0}=0, h_{1}=2, h_{2}=8$, $h_{3}=36, h_{4}=154, \ldots$

Our second recursion is generated by hole dynamics run until growth from the initial two buds collides:

$$
h_{n}^{k}=2 b_{n-1}+h_{n-1}^{k}+h_{n-1}^{k+1}-e_{k}, \quad 2^{n-1} \geq 4 k+8 .
$$

The restriction on $n$, obtained from Lemma 4.1 below, is not optimal, but is one that works in all cases. (For small $k$ one can get away with a less restrictive condition, which varies from case to case and is useful for computations.) The error terms $e_{k}$, which keep this recursion from closing, are the result of slightly "dirty" interaction between the two growing buds (cf. the bottom of Fig. 4).

We bound $e_{k}$ using the following fact about additive dynamics. Starting from $T_{0}=\{0\}$, perform $2^{n}-1$ steps to generate $T_{2^{n}-1}$. Fix an $\ell, 0<\ell \leq 2^{n}$ and let $Z=Z(n, \ell)$ be the union of all connected components (in the triangular lattice sense) of 0's in $T_{2^{n}-1} \cap\left\{(x, y): y \geq 2^{n}-\ell\right\}$.

Lemma 4.1. Every $(x, y) \in Z$ has $y \geq 2^{n}-2 \ell$.

Proof. Focus on the line $\left\{y=2^{n}-\ell\right\}$ and consider an interval of $a$ 0's flanked by 1's at both ends. Find the first 1 below, say at distance $b$, the leftmost 0 of this interval. Paint this occupied site red. Then the column of $b+1$ sites ( $b 0$ 's and the red site) must have to its immediate left a column of $b+11$ 's. The dynamics now ensures that $a=b$ and that the red site is connected by an occupied diagonal to the 1 at the right border of the initial interval of 0's.

This proves that the worst case is when $T_{2^{n}-1} \cap\left\{y=2^{n}-\ell\right\}$ consists of $\ell-10$ 's flanked by 21 's, in which case $b=\ell-1$.

One can in principle compute $e_{k}$ for any $k$ from quantities $e_{k}^{w}$ and $e_{k}^{h}$ which we now define.

\begin{tabular}{|c|c|c|c|c|c|c|c|c|}
\hline \multirow[t]{3}{*}{1} & 1 & 1 & 1 & 1 & $x$ & $x$ & $x$ & $x$ \\
\hline & & & & $x$ & $x$ & $x$ & $x$ & $x$ \\
\hline & & & $x$ & $x$ & $x$ & $x$ & $x$ & $x$ \\
\hline \multirow{5}{*}{$x$} & & $x$ & $x$ & $x$ & $x$ & $x$ & $x$ & $x$ \\
\hline & $x$ & $x$ & $x$ & $x$ & $x$ & $x$ & $x$ & $x$ \\
\hline & $x$ & $x$ & $x$ & $x$ & 1 & $x$ & $x$ & $x$ \\
\hline & & & & 1 & & $x$ & $x$ & \\
\hline & & & 1 & & & $x$ & & \\
\hline
\end{tabular}
First, $e_{k}^{w}$ is the final occupation count in the region at the tip of the growth that gives $b_{n}$, consisting of a $(2 k+3) \times(2 k+3)$ box together with two lattice triangles, a $(2 k+3) \times(2 k+3)$ and a $(2 k+1) \times(2 k+1)$ one. Here is the region for $k=1$, labeled with $x$ 's: 
Next, $e_{k}^{h}$ is the final count of occupied sites in the interaction area in the middle of $H\left(2^{n}-2 k\right)$. This area consists of a $(2 k+3) \times(2 k+3)$ box and four triangles - the two above and their reflections across the main diagonal of the box. (The set of occupied sites is symmetric with respect to this reflection at all times.)

In all small cases we use, we have checked that the last occupied site in the interaction area (or in fact anywhere outside the two smaller holes that are filled recursively) gets added at time $2^{n-1}$. If this is the case, a speed of light argument shows that $e_{n}^{w}$ can be computed in the $(2 k+3) \times(2 k+3)$ box with an additional row of $2 k+3$ sites at the bottom, while the interaction area for computation of $e_{k}^{w}$ adds to this an additional column of $2 k+3$ sites on the right. Then (4.3) holds under the restriction $2^{n-1} \geq 2 k+6$. As mentioned earlier, we use this property of small cases for computations.

In any case, by Lemma 4.1 above (and its proof),

$$
e_{k}=2 e_{k}^{w}-e_{k}^{h}
$$

Direct enumeration gives $e_{-1}=2, e_{0}=10, e_{1}=24, e_{2}=44, e_{3}=66, e_{4}=92, e_{5}=128$, $e_{6}=170, e_{7}=212, e_{8}=258$.

Observe that if we know $h_{n}^{K}, n \leq N$, and $e_{k}, k \leq K-1$, then we can use (4.2) and (4.3) to compute $b_{n}$ up to $n \leq N+K+3$.)

An explicit formula for $e_{k}$ is apparently too much to hope for, but using the trivial facts that $e_{k}^{h}$ and $e_{k}^{w}$ are nonnegative and bounded above by the number of sites in their respective regions, we do have the bounds

$$
-3(2 k+3)^{2}<-e_{k}^{h} \leq e_{k} \leq 2 e_{k}^{w}<4(2 k+3)^{2} .
$$

Write

$$
\eta(n)=\sum_{k=0}^{n-1} \sum_{i=0}^{k}\left(\begin{array}{l}
k \\
i
\end{array}\right)(2 i+1)^{2}=n^{2} 2^{n}-n 2^{n}+2^{n}-1 .
$$

Using (4.3) repeatedly, and the above bounds, one obtains

$$
h_{n}=\sum_{k=1}^{n} 2^{k} b_{n-k}-e_{n}^{\prime} .
$$

where

$$
-3 \eta(n) \leq e_{n}^{\prime} \leq 4 \eta(n)
$$

Therefore

$$
\begin{aligned}
b_{n+1} & =3 b_{n}+2 b_{n-1}+2 \sum_{k=1}^{n-1} 2^{k} b_{n-1-k}-e_{n-1}^{\prime}-2^{n+1}+4 \\
& =3 b_{n}+\sum_{k=1}^{n} 2^{k} b_{n-k}-e_{n}^{\prime \prime},
\end{aligned}
$$

where $e_{n}^{\prime \prime}=e_{n-1}^{\prime}+2^{n+1}-4$ for $n \geq 1$ and $e_{0}^{\prime \prime}=-1$. 
The sequence $b_{n} / 4^{n}$ satisfies a renewal equation, and the renewal theorem ([Fel, p. 330]) gives

$$
\rho_{1}=\lim _{n \rightarrow \infty} \frac{b_{n}}{4^{n}}=\frac{1-\frac{1}{4} \sum_{n=0}^{\infty} 4^{-n} e_{n}^{\prime \prime}}{\frac{3}{4}+\frac{1}{2} \sum_{k=2}^{\infty} k 2^{-k}}=\frac{5}{6}-\frac{1}{6} \sum_{n=1}^{\infty} 4^{-n} e_{n}^{\prime \prime} .
$$

Summability of $4^{-n} e_{n}^{\prime \prime}$ guarantees existence of the limit, but $\rho_{1}$ could be 0 or 1 in principle.

To obtain nontrivial bounds on $\rho_{1}$, we enumerate $h_{n}^{9}$ for $n \leq 11$ directly, employ (4.2) and (4.3) to compute $b_{n}$ exactly for $n \leq 23$, and then use (4.5) to compute $e_{n}^{\prime \prime}$ for $n \leq 22$. For $n \geq 23$, we estimate $e_{n}^{\prime \prime}$ as follows.

The lack of symmetry between the upper and lower bounds in (4.4) can be removed by counting 0's instead of 1's. That is, write $b_{n}^{c}=2^{n}\left(2^{n}+2\right)-b_{n}$ and $h_{n}^{c}=\left(2^{n}+1\right)^{2}-h_{n}$. Then the analogues of (4.2) and (4.3) lead to

$$
b_{n+1}^{c}=3 b_{n}^{c}+\sum_{k=1}^{n} 2^{k} b_{n-k}^{c}-e_{n-1}^{\prime}-4 \cdot 2^{n}-6 .
$$

Although these $e_{n}^{\prime}$ are different from the previous ones, we do not introduce new notation, because they satisfy the same bounds (4.4). Changing (4.7) into an equation for $b_{n}$, and using the result to obtain the upper bound for $e_{n}^{\prime \prime}$ in (4.5), we get

$$
-3 \eta(n-1)+2^{n+1}-4 \leq e_{n}^{\prime \prime} \leq 3 \eta(n-1)+2 n \cdot 2^{n}-3 \cdot 2^{n}-6 .
$$

The dominant term in both upper and lower bound is $3 n^{2} 2^{n}$. This can in fact be improved to $\frac{5}{2} n^{2} 2^{n}$, as a better bound for $e_{k}$ is obtained by considering where the two wedge dynamics and the hole dynamics must agree. A little geometric argument demonstrates that this agreement is achieved at least within a region between lines of slope 2 and $1 / 2$ through the center of the interaction region. We omit the details, as it is much easier to improve the bounds on $\rho_{1}$ by computing more $e_{k}$ 's for small $k$ than by trying to improve (4.8).

Our computations yield $4^{-23} b_{23} \approx 0.635280$, and so, using (4.6) and (4.8), the rigorous bounds

$$
0.635248 \leq \rho_{1} \leq 0.635312 .
$$

Our second rule is Hex 14. With minor changes our analysis also applies to Hex 145, so we will omit that case.

The first basic recursion now reads, for $n \geq 3$,

$$
w_{n+1}=3 w_{n}+2 w_{n-1}+h_{n-1}-2 \cdot 2^{n}-2,
$$

and this time

$$
b_{n}=2 w_{n}+2^{n} .
$$

This yields, with corrections computed separately for low $n$,

$$
b_{n+1}=3 b_{n}+2 b_{n-1}+2 h_{n-1}-6 \cdot 2^{n}-4-1_{\{n=1\}}+2 \cdot 1_{\{n=2\}},
$$

for $n \geq 1$. We have $b_{0}=3, b_{1}=8, b_{2}=24, b_{3}=78, b_{4}=280, \ldots$, and $h_{0}=4, h_{1}=8, h_{2}=25$, $h_{3}=80, \ldots$ The second recursion remains (4.3). 
What does change in this case is computational. Namely, in all small cases we use for our estimates, the computation for $b_{n}$ ends at time $2^{n}+1$, and at time $2^{n-1}+1$ in the interaction area. For computation of $e_{k}^{h}$ this forces us to add two layers of $2 k+3$ sites at the bottom and on the right of the central $(2 k+3) \times(2 k+3)$ box and one layer of $2 k+5$ sites at the top and on the left of that box. For computation of $e_{k}^{w}$, the added layers are of course only at the bottom and on the left, creating a $(2 k+5) \times(2 k+4)$ box. (The restriction in (4.3) then is $2^{n-1} \geq 2 k+8$.) In this way, we get $e_{-1}=2, e_{0}=11, e_{1}=27, e_{2}=49, e_{3}=82, e_{4}=125$, and $e_{5}=170$.

Thus

$$
b_{n+1}=3 b_{n}+\sum_{k=1}^{n} 2^{k} b_{n-k}-e_{n}^{\prime \prime}
$$

where $e_{n}^{\prime \prime}=e_{n-1}^{\prime}+6 \cdot 2^{n}+4+1_{\{n=1\}}-2 \cdot 1_{\{n=2\}}$ for $n \geq 1, e_{0}^{\prime \prime}=1$, and $e_{n}^{\prime}$ satisfies the same bounds (4.4). In this case the renewal theorem gives

$$
\rho_{14}=\lim _{n \rightarrow \infty} \frac{b_{n}}{4^{n}}=\frac{3-\frac{1}{4} \sum_{n=0}^{\infty} 4^{-n} e_{n}^{\prime \prime}}{\frac{3}{4}+\frac{1}{2} \sum_{k=2}^{\infty} k 2^{-k}}=\frac{11}{6}-\frac{1}{6} \sum_{n=1}^{\infty} 4^{-n} e_{n}^{\prime \prime} .
$$

The estimates of $e_{n}^{\prime \prime}$ for large $n$ are as follows (this time we do not bother to symmetrize):

$$
-3 \eta(n-1)+6 \cdot 2^{n}+4 \leq e_{n}^{\prime \prime} \leq 4 \eta(n-1)+6 \cdot 2^{n}+4 .
$$

For this case we compute $b_{n}$ up to $n=20$, to get $4^{-20} b_{20} \approx 0.968854$ and

$$
0.968618<\rho_{14}<0.969044 \text {. }
$$

Next, we turn to Hex 16 .

All definitions, as well as recursions (4.2) and (4.3), remain exactly the same as for Hex 1. Note that the "6" part of the rule only influences sites that have no further influence elsewhere, so this rule has exactly the same interactions as Hex 1 . For example, we can obtain $A_{\infty}$ for $H e x$ 1 and then perform a single Hex 16 step to obtain $A_{\infty}$ for $\operatorname{Hex} 16$.

The extra step does affect the computation of $e_{k}$, making in small cases the interaction areas the same as for the Hex 14 rule. To repeat: for $e_{k}^{h}$ we add two layers of $2 k+3$ sites at the bottom and on the right of the central $(2 k+3) \times(2 k+3)$ box and one layer of $2 k+5$ sites at the top and on the left of that box, while for $e_{k}^{w}$ the added layers are only at the bottom and on the left. The restriction in (4.3) is $2^{n-1} \geq 2 k+8$.

We get $b_{0}=1, b_{1}=4, b_{2}=16, b_{3}=58, b_{4}=212$, etc., and $e_{-1}=4, e_{0}=15, e_{1}=32$, $e_{2}=56, e_{3}=83, e_{4}=115$, and $e_{5}=157$. By computing $h_{n}^{6}$ up to $n=9$, we obtain $2^{-18} b_{18} \approx 0.739664$ and

$$
0.738902<\rho_{16}<0.740279
$$

Next in line is Hex 156.

The first recursion now is similar to (4.9);

$$
b_{n+1}=3 b_{n}+2 b_{n-1}+2 h_{n-1}-6 \cdot 2^{n}+10-6 \cdot 1_{\{n=1\}},
$$

while (4.3) is still the second recursion. In addition, $b_{n}=2^{n}\left(2^{n}+2\right)$ up to $n=3$; after that permanently empty triangles (of 6 sites) appear. Much later (as per the discussion in Section 8), larger permanently empty regions appear. 
In this case, two extra steps are required (again, for the small cases we have checked) to finalize the configuration in $B_{n}$. The last of these two steps, however, merely fills two sites next to the axes and thus does not affect any computations. The interaction areas are therefore the same as for Hex 16.

This time, we get $b_{0}=3, b_{1}=8, b_{2}=24, b_{3}=80, b_{4}=276$, etc., and $e_{-1}=1, e_{0}=9$, $e_{1}=25, e_{2}=55, e_{3}=84, e_{4}=106$, and $e_{5}=151$. By computing $h_{n}^{6}$ up to $n=9$, we obtain $2^{-18} b_{18} \approx 0.937935$ and

$$
0.937183<\rho_{16}<0.938559 .
$$

Our last rule in this section is Hex 15. In this case, the first recursion is

$$
b_{n+1}=3 b_{n}+2 b_{n-1}+2 h_{n-1}-6 \cdot 2^{n}+8-6 \cdot 1_{\{n=1\}},
$$

and we get $b_{0}=3, b_{1}=8, b_{2}=22, b_{3}=70, b_{4}=238$, etc., and $e_{-1}=0, e_{0}=6, e_{1}=18, e_{2}=42$, $e_{3}=66, e_{4}=84$, and $e_{5}=120$. By computing $h_{n}^{6}$ up to $n=9$, we obtain $2^{-18} b_{18} \approx 0.802578$ and

$$
0.801822<\rho_{16}<0.803199 .
$$

We note that again small cases require two steps to finalize the configuration in $B_{n}$. Both steps fill two sites next to the axes and are thus not problematic. The new feature is different hole dynamics, requiring one extra step (beyond $2^{n-1}$ ) to resolve. Therefore the interaction areas are the same as for Hex 16.

\section{Density of Hex 1456 and Hex 146}

For concreteness, we will concentrate on Hex 1456. The same techniques applied to Hex 146 yield the bounds in Theorem 1.2. The definitions of $b_{n}$ and $h_{n}$ are the same as in Section 3. The first recursion now is

$$
b_{n+1}=3 b_{n}+2 b_{n-1}+2 h_{n-1}-6 \cdot 2^{n}-2,
$$

while the second is still (4.3). We note that, if $h_{n} \equiv\left(2^{n}+1\right)^{2}$, then $b_{n} \equiv 2^{n}\left(2^{n}+2\right)$. That is, if every hole fills completely, then $A_{n}$ fills the lattice and $\rho_{1456}=1$. At first, simulations suggest that this indeed happens.

However, after more systematic experimentation one discovers that $H(82)$ and $H(84)$ do not fill completely; a final triangle of 0's consists of 25 sites. For the lower bound, it is also important to observe that all the other holes of sizes at most 130 do fill in. Therefore, all boxes of sizes $2^{n}-44, n \geq 7$ do not fill in. (In fact, they leave $2(n-7)+1$ unfilled triangles of size 25 .) Since there are infinitely many such boxes generated by the dynamics, the number of unfilled 0 's is infinite. This however does not establish that $\rho_{1456}<1$. In fact, we do not even have a nontrivial lower bound for the density yet, so we start with this easier task.

A zero-creating hole will occur for the first time inside a $H\left(2^{n}+2\right)$ when $2^{n-22}-44 \geq 84$, hence $n \geq 28$. Thus $b_{n}=2^{n}\left(2^{n}+2\right)$ for $n \leq 29$. (This explains why a naive simulation started with a single occupied site will never produce a hole - one would need a system of a size more than $2^{30} \approx 10^{9}$.)

As in (4.7), let $b_{n}^{c}=2^{n}\left(2^{n}+2\right)-b_{n}$ and $h_{n}^{c}=\left(2^{n}+1\right)^{2}-h_{n}$. Then

$$
b_{n+1}^{c}=3 b_{n}^{c}+2 b_{n-1}^{c}+2 h_{n-1}^{c}
$$


and $h_{n}^{c}$ satisfy the analogue of (4.3), with the same bounds (4.4) on $e_{k}$. Hence

$$
b_{n+1}^{c}=3 b_{n}^{c}+\sum_{k=1}^{n} 2^{k} b_{n-k}^{c}-e_{n-1}^{\prime},
$$

where $e_{n}^{\prime}=0$ for $n \leq 28$. Furthermore, $e_{n}^{\prime}$ satisfies the bounds (4.4). The renewal theorem therefore guarantees the existence of a density, and, by (4.4),

$$
1-\rho_{1456}=\lim _{n \rightarrow \infty} \frac{b_{n}^{c}}{4^{n}}=-\frac{1}{24} \sum_{n=29}^{\infty} 4^{-n} e_{n}^{\prime}<\frac{1}{8} \sum_{n=29}^{\infty} 4^{-n} \cdot n^{2} 2^{n}<\frac{28^{2}}{8 \ln 2 \cdot 2^{28}}<5.3 \cdot 10^{-7} .
$$

Unfortunately, a nontrivial upper bound seems very difficult to produce by a brute force approach along the lines of Section 4. Instead, we devise a rescaling argument.

Let $R$ be a time at which there is a permanent 0 in $A_{R}$ (with the initial condition that produces $b_{n}$ ). Clearly we can choose $R$ to be a power of 2 , and arbitrarily large. We can also ensure that the 0 is at distance at least $R / 2$ (in $\|\cdot\|_{\infty}$ distance, say) away from the part of the boundary of $A_{R}$ strictly inside the first quadrant.

Now run the dynamics in time steps of size $R$, with the proviso that, whenever a hole is formed, the clock inside this hole is reset so that time 0 corresponds to the formation of the hole. The dynamics then uses different clocks on different regions of the plane. The point is that every second time, starting with time 1, every added occupied site is part of a version of $A_{R}$ (its translation and/or rotation). The other times are used for filling in, and holes cannot be guaranteed.

As we know, the buds inside the holes interact, but the interaction will not reach the existing 0 until it is of size $R / 2$, therefore up to (unrescaled) time at least $2^{R / 4}$. Up to that time, therefore, all the occupied sites will see a permanent 0 within distance $4 R$.

Next, let $d_{n}$ be the site count of sites within distance $4 R$ of a permanent 0 , at time $2^{n}+1$. As the interaction can destroy a 0 and therefore affect sites at distance $4 R$ from the interaction area, $d_{n}$ satisfy the same recursion as $b_{n}$, but with error bounds multiplied by $81 \cdot R^{2}$. If $d_{n}^{c}=2^{n}\left(2^{n}+2\right)-d_{n}$, then

$$
d_{n+1}^{c}=3 d_{n}^{c}+\sum_{k=1}^{n} 2^{k} d_{n-k}^{c}-e_{n-1}^{\prime}
$$

where in this version

$$
\left|e_{n}^{\prime}\right|<10^{3} R^{2} n^{2} 2^{n} .
$$

Moreover, we already know that $e_{n}^{\prime}=0$ for $n \leq R / 4$. Therefore

$$
\limsup \frac{d_{n}^{c}}{4^{n}}<\sum_{n \geq R / 2} 10^{3} R^{2} n^{2} 2^{-n}
$$

which clearly can be made arbitrarily small for large enough $R$.

It follows that a positive proportion of occupied sites sees a permanent 0 within a fixed distance. Therefore the final density of 1's is necessarily strictly less than 1 . 


\section{Proof of Theorem 1}

We start with the case $A_{0}=\{0\}$. What we need to prove, roughly, is that the proportion of $A_{\infty}$-sites in any nice large subset of $\mathbb{Z}^{2}$ is about $\rho$. We accomplish this by the division of space into "mesoscopic" units filled in by the basic wedge dynamics (which gives $w_{n}$ ) and much smaller problematic regions.

To this end, let us return to the recursion for $w_{n}$. This recursion can be "unrolled." That is, when not both $\pi(3)$ and $\pi(4)$ are $1, w_{n}$ can be written in terms of $w_{n-m-1}, \ldots, w_{n-m-\ell}$, and $h_{n-m-\ell}^{k}$, for some $m \geq 0, \ell \geq 2$, and suitable $k$ 's. This is the case we will focus on for the remainder of the proof. When $\pi(3)=\pi(4)=1, h_{n-m-\ell}^{k}$ is replaced by a suitable linear combination of $h_{n-m-\ell}, s_{n-m-\ell}$ and $t_{n-m-\ell}$, and the proof is easily adapted. (The right side of Fig. 5 illustrates the difference between resulting divisions.) The unrolled recursion corresponds to a division of $W_{n}$ into smaller triangles and parallelograms. If $n$ is large, and $W_{n}$ is normalized by $1 / 2^{n}$, this partition approximates a partition of the unit triangle $W=$ $\{(x, y): 0 \leq y \leq x \leq 1\}$. In this way, $W$ is divided into covering triangles (corresponding to $w_{i}$ ) and parallelograms. We define these sets to be closed, so there is some intersection along boundaries. Here, $m$ determines the largest size of a triangle used, which is $1 / 2^{m+1}$ times the size of $W$. Furthermore, $\ell$ gives exactly the number of different triangle sizes used. The left side of Fig. 5 pictures the division for $m=1$ and $\ell=3$, with the remaining parallelograms shaded. The maximum diameter of sets in the division clearly halves with each successive $m$. Moreover, the area covered by the parallelograms (uncovered by triangles) halves with each successive $\ell$. View $m$ and $\ell$ as large, but much smaller than $n$.
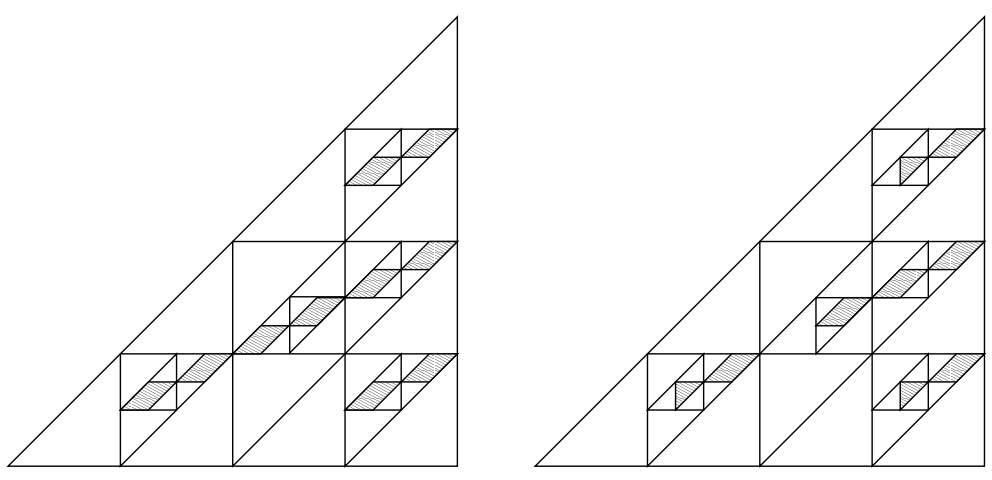

Fig. 5. The division of $W$ for $m=1$ and $\ell=3$ in two cases.

Fix an $\epsilon>0$. It is clear that $\left|4^{-(n-m-i)} w_{n-m-i}-\frac{1}{2} \rho\right|, i=1, \ldots, \ell$, can be made simultaneously smaller than $\epsilon$ if $n$ is chosen large enough. (Note that $\frac{1}{2} \cdot 4^{-(n-m-i)}$ is exactly the area of the corresponding triangle in the division.)

Pick a closed set $S \subset W$, with nice boundary. For example, we may assume that $S$ is convex and $\partial S$ consists of finitely many linear pieces. How many points from $2^{-n}\left(A_{\infty} \cap W_{n}\right)$ does $S$ contain? In the sequel, $C$ is a "generic constant," which is allowed to vary from one appearance to another.

First, the triangles that intersect $\partial S$ together contain at most $C \cdot \operatorname{length}(\partial S) \cdot 2^{2 n-m}$ points. Second, all the parallelograms in $W$ have combined area at most $C \cdot 2^{-\ell}$ and so they contain at most $C \cdot 2^{2 n-\ell}$ points. A crude upper bound on the total length of the boundaries of the 
division sets is 4 times the number of division sets, which can be (equally crudely) bounded by the reciprocal of the area of the smallest set in the division. Therefore, the total boundary length is at most $C \cdot 4^{m+\ell}$ and so within (microscopic) distance $C$ of these boundaries there are at most $C \cdot 2^{n+2 m+2 \ell}$ points. Finally, we know that the error terms (if any) contribute at most $C \cdot n^{2} \cdot 2^{n}$ points.

Therefore, $S$ contains at least

$$
\left((\rho-\epsilon) \cdot \operatorname{area}(S)-C \cdot \operatorname{length}(\partial S) \cdot 2^{-m}-C \cdot 2^{-\ell}-C \cdot 2^{-n+2 m+2 \ell}-C \cdot n^{2} \cdot 2^{-n}\right) \cdot 4^{n}
$$

points, for large enough $n$. An analogous upper bound also holds. By letting first $n \rightarrow \infty$, then $m, \ell \rightarrow \infty$, and finally $\epsilon \rightarrow 0$, it follows that

$$
\frac{1}{4^{n}}\left|S \cap 2^{-n}\left(A_{\infty} \cap W_{n}\right)\right| \rightarrow \rho \cdot \operatorname{area}(S) .
$$

The proof of Theorem 1 for $A_{0}=\{0\}$ is now concluded in a straightforward manner.

For general finite $A_{0}$, Theorem 1 mostly follows from properties of additive dynamics, as we explain below. The proof is somewhat delicate, which is not a complete surprise since sensitivity to perturbations in initial condition is widespread among generic cellular automaton rules. In fact, there is a "box snowflake" for which the density of $A_{\infty}$ has been proved to depend on the initial set. Namely, we have shown in [GG1] that Box 1 solidification yields density 4/9 starting from a singleton. Later, Dean Hickerson [Hic] engineered finite initial seeds with asymptotic densities $29 / 64$ and $61 / 128$. For instance, the latter is achieved by an ingenious arrangement of 180 carefully placed occupied cells around the boundary of an $83 \times 83$ grid. We do not know the highest density with which Box 1 solidification can fill the plane, nor whether any seed fills with density less than $4 / 9$.

To return to hexagonal digital snowflakes, we will assume that $A_{0}$ has sites on the $y$-axis, the lowest of which is at 0 and the highest at $(0, h)$, but no sites to the right of $y$-axis. We will concentrate on proving the density result for the part of $A_{\infty}$ between lines $y=0$ and $y=x+h$, which, by symmetry and the extreme boundary dynamics, is clearly enough. We start with the following fact about additive dynamics. Assume the seed is $T_{0}=A_{0} \cap y$-axis. By additivity, when $2^{n}-1>h$, the rightmost column of $T_{2^{n}}$ consists of two copies of $T_{0}$, separated by $2^{n}-1-h$ 0 's. The column immediately to the left of these 0's either consists of all 1's (flanked by two 0 's) or else of all 0's, flanked on the top by a 1 and on the bottom by an additional 0, below which there is a 1 . In the second case we can iterate and continue moving to the left until we encounter a column of 1 's (flanked by a 0 on both ends). Assume this column consists of $D$ 1's. An important point is

$$
2^{n}-h \leq D \leq 2^{n} .
$$

Furthermore, if this column is created (by the boundary dynamics) at time $t$, then at time $t+D+1$ the boundary dynamics creates a horizontal and a diagonal segment which together with the above column seal off a triangle of 0's. Into this triangle (which is a bounded perturbation of the one started from a singleton), the Hex dynamics spread as when they fill basic wedges.

However, interaction between the secondary boundary dynamics is slightly more complicated. When the boundary dynamics from the two secondary buds (cf. Section 2) collide, they (together with the primary boundaries) create a hole which is equal or smaller in size to that from $A_{0}=\{0\}$. In fact the hole's side can be diminished by at most $h-1$. By Lemma 4.1 the hole generates smaller holes (all of which are smaller, and bounded perturbations of the $A_{0}=\{0\}$ 
case), provided all the wedge vertices that were dead before are still dead. The problematic vertices are created in the middle of the holes when secondary boundary dynamics collide. If $\pi(3)$ and $\pi(4)$ are not both 1 , collisions always result in dead vertices, as is easy to check. We will deal with this case first.

Pick a closed convex set $S \subset W$ with piecewise linear boundary and an $\epsilon>0$. In the first step, perform $m$ steps of the Sierpinski triangle construction. That is, take out of $W$ the central triangle congruent to $W / 2$, then 3 triangles congruent to $W / 4, \ldots$, and finally $3^{m}$ triangles congruent to $W / 2^{m}$. Discard the points in $W$ outside these triangles (see top left of Fig. 6 for the $m=3$ example). In the second step, mark by $\mathrm{M}$ the two smaller triangles from each triangle obtained in the first step, as in Fig. 6. In the third step, mark 4 triangles in the remaining quadrilateral hole, then 4 triangles in each of the remaining two holes, and so on, for a total of $m$ iterations and $4 \cdot 2^{m}$ triangles. The top of Fig. 6 illustrates these three steps for $m=3$. Note that when $m=m(\epsilon)$ is large enough, the area of the exceptional set, i.e., the set of points outside of marked triangles is below $\epsilon / 4$. For a small enough $\delta=\delta(\epsilon, m)$, the area of $W_{e}$, the $\delta$-neighborhood of the exceptional set, is below $\epsilon / 2$.

Now, take a large $n$ and consider $2^{-n} A_{\infty} \cap W$ on one of the marked triangles $W_{M}$, off $W_{e}$. By Lemma 2.1, this configuration exactly equals the configuration of sites in a basic wedge if $n \geq n_{0}(\delta)$. Therefore, by taking $n$ even larger if necessary,

$$
\left|2^{-n} A_{\infty} \cap W_{M} \cap W_{e}^{c} \cap S\right| \geq \rho \cdot \operatorname{area}\left(W_{M} \cap W_{e}^{c} \cap S\right)-6^{-m-1} \epsilon,
$$

by the already proved result for basic wedges. Since the number of marked triangles is less than $2 \cdot 6^{m}$, it follows that

$$
\left|2^{-n} A_{\infty} \cap S\right| \geq \rho \cdot \operatorname{area}(S)-\epsilon,
$$

for $n \geq n_{0}(\epsilon)$, as desired. The analogous upper bound is proved in the same fashion.

When $\pi(3)=\pi(4)=1$, then the middle vertices in the holes are live as soon as $D<2^{n}$. Although $h>0$ does not necessarily imply that $D<2^{n}$, there is for each $h$ a unique $T_{0}$ which has $D=2^{n}$, namely the one generated by the additive dynamics starting from $\{0\}$ and run for $h$ time steps (and translated back so that its lowest site is at 0 ).

In this case, therefore, the division into marked triangles in step 3 has two possible forms, one for $D=2^{n}$ and one for $D<2^{n}$. Both are indicated in the bottom of Fig. 6. In either case, the proof is then a minor modification of the above.

This concludes the proof of Theorem 1 . To finish this section, we assume $\pi(3)=0$ and prove (4.1), or equivalently, that for the dynamics on $W_{n}$,

$$
4^{-n}\left|A_{2^{n}-1} \cap W_{n}\right| \rightarrow \frac{1}{2} \rho .
$$

In fact, the recursion for $w_{n}$ can be used to show that $A_{2^{n}+C n^{2}} \cap W_{n}=A_{\infty} \cap W_{n}$, since the largest interaction area inside the holes is on the order of $n^{2}$. (It is possible that in all cases $A_{2^{n}+c} \cap W_{n}=A_{\infty} \cap W_{n}$, for some small constant $c$, but we cannot prove this.) Then, in the division as in the case $A_{0}=\{0\}$ above, only the dynamics inside triangles that intersect the right edge of $W$ is not done by time $2^{n}$. These contain at most $C \cdot 2^{2 n-m}$ points and the proof is concluded as before. (Growth inside some parallelograms might also persist, but such sites are already incorporated into the error.) 


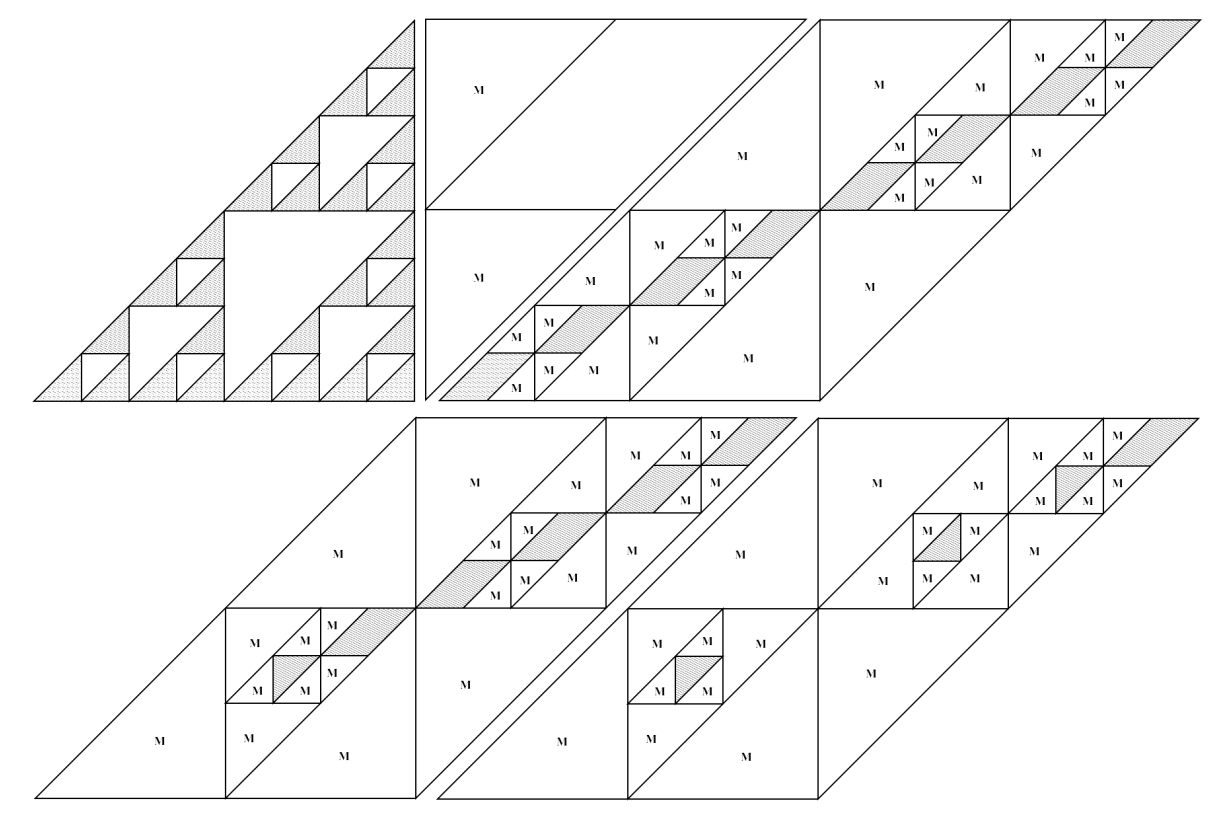

Fig. 6. The three steps and marked triangles for $m=3$. Regions outside them are shaded.

\section{$7 \quad$ Macroscopic dynamics}

In this section we assume $A_{0}=\{0\}$ and study $A_{t}$ for a large finite $t$ instead of $A_{\infty}$. As the radius of $A_{t}$ increases linearly in $t$, it is natural to ask whether these dynamics have a hydrodynamic limit as space and time are scaled proportionally. Computer simulations certainly suggest so, and are validated by our analysis below.

There are two kinds of macroscopic dynamics for digital snowflakes, both denoted here by $\mathcal{S}_{a}, a \in[0,1]$. The sets $\mathcal{S}_{a}$ will be a strictly increasing family of closed subsets of the hexagon $\operatorname{co}(\mathcal{N}) \subset \mathbb{R}^{2}$. We start with the simpler case, which we call Simple Hole Dynamics (SHD). This evolution will be associated with the 12 rules for which $\pi(3)$ and $\pi(4)$ are not both 1 .

Assume $a<1$ and write the dyadic expansion $a=0 . a_{1} a_{2} \ldots$ with infinitely many 0's. We denote $a^{\prime}=0 . a_{2} a_{3} \ldots, a^{\prime \prime}=\left(a^{\prime}\right)^{\prime}$, etc., and use the following transformations: reflection $\sigma$ about the line $y=x$, rotation $\rho_{\alpha}$ by an angle $\alpha$, and deformation $\delta$ by the linear transformation $(x, y) \mapsto(x-y, y)$. We will only define the portion of $\mathcal{S}_{a}$ inside the triangle $W=\left\{(x, y) \in \mathbb{R}^{2}\right.$ : $0 \leq x \leq y \leq 1\}$; other parts are then obtained by symmetry, i.e., by an appropriate use of the above transformations.

We will first recursively define $\mathcal{S}_{a} \subset W$, and a corresponding hole dynamics $\mathcal{H}_{a} \subset W$ on dyadic rationals $a$ that have only finitely many 1's in their dyadic expansion, starting with $\mathcal{S}_{a}=\{(0,0)\}$ and $\mathcal{H}_{a}=\emptyset$ for $a=0$. The rules described in the next paragraph and Fig. 7 are a natural extension of the recursions for $w_{n}$ and $h_{n}$ for the corresponding 12 rules. (We remark that when no two successive $a_{i}$ 's are 1 , one can define the boundary of $\mathcal{S}_{a}$ by a Kochtype substitution scheme, as outlined in the Introduction for $a=1 / 3$ and in [GG1] for Box 1 solidification; such algorithms are in general precluded by collisions inside the holes.) 
If $a_{1}=0$, then take $\mathcal{S}_{a}=\frac{1}{2} \mathcal{S}_{a^{\prime}}$. If $a_{1}=1$ and $a_{2}=0$, then $S_{a}$ consists of five pieces:

$$
\begin{aligned}
& \mathcal{S}_{a}=\frac{1}{2} W \quad \cup \quad\left(\frac{1}{2}, 0\right)+\frac{1}{2} \mathcal{S}_{a^{\prime}} \cup\left(\frac{1}{2}, \frac{1}{2}\right)+\frac{1}{2} \mathcal{S}_{a^{\prime}} \\
& \cup\left(\frac{1}{2}, 0\right)+\frac{1}{4} \sigma \mathcal{S}_{a^{\prime \prime}} \cup\left(\frac{1}{2}, \frac{1}{2}\right)+\frac{1}{4} \rho_{-\pi / 2} \delta \mathcal{S}_{a^{\prime \prime}} .
\end{aligned}
$$

If $a_{1}=1$ and $a_{2}=1$, then $S_{a}$ consists of seven pieces:

$$
\begin{array}{rllllll}
\mathcal{S}_{a}=\frac{1}{2} W & \cup & \left(\frac{1}{2}, 0\right)+\frac{1}{2} \mathcal{S}_{a^{\prime}} & \cup & \left(\frac{1}{2}, 0\right)+\frac{1}{2} \mathcal{S}_{a^{\prime}} & \cup & \left(\frac{1}{2}, \frac{1}{2}\right)+\frac{1}{4} \sigma W \\
& \cup & \left(\frac{1}{2}, \frac{1}{2}\right)+\frac{1}{4} \rho_{-\pi / 2} \delta W & \cup & \left(\frac{1}{2}, \frac{1}{4}\right)+\frac{1}{4} \mathcal{H}_{a^{\prime \prime}} & \cup & \left(1, \frac{1}{2}\right)+\frac{1}{4} \rho_{\pi} \mathcal{H}_{a^{\prime \prime}} .
\end{array}
$$

If $a_{1}=0$, then

$$
\mathcal{H}_{a}=(1,1)+\frac{1}{2} \rho_{\pi} \sigma \mathcal{S}_{a^{\prime}} \quad \cup \quad(1,0)+\frac{1}{2} \rho_{\pi / 2} \delta \mathcal{S}_{a^{\prime}}
$$

and if $a_{1}=1$,

$$
\mathcal{H}_{a}=(1,1)+\frac{1}{2} \rho_{\pi} \sigma W \quad \cup \quad(1,0)+\frac{1}{2} \rho_{\pi / 2} \delta W \quad \cup \quad \frac{1}{2} \mathcal{H}_{a^{\prime}} \quad \cup \quad\left(1, \frac{1}{2}\right)+\frac{1}{2} \rho_{\pi} \mathcal{H}_{a^{\prime}} .
$$

$\mathcal{S}_{a}:$
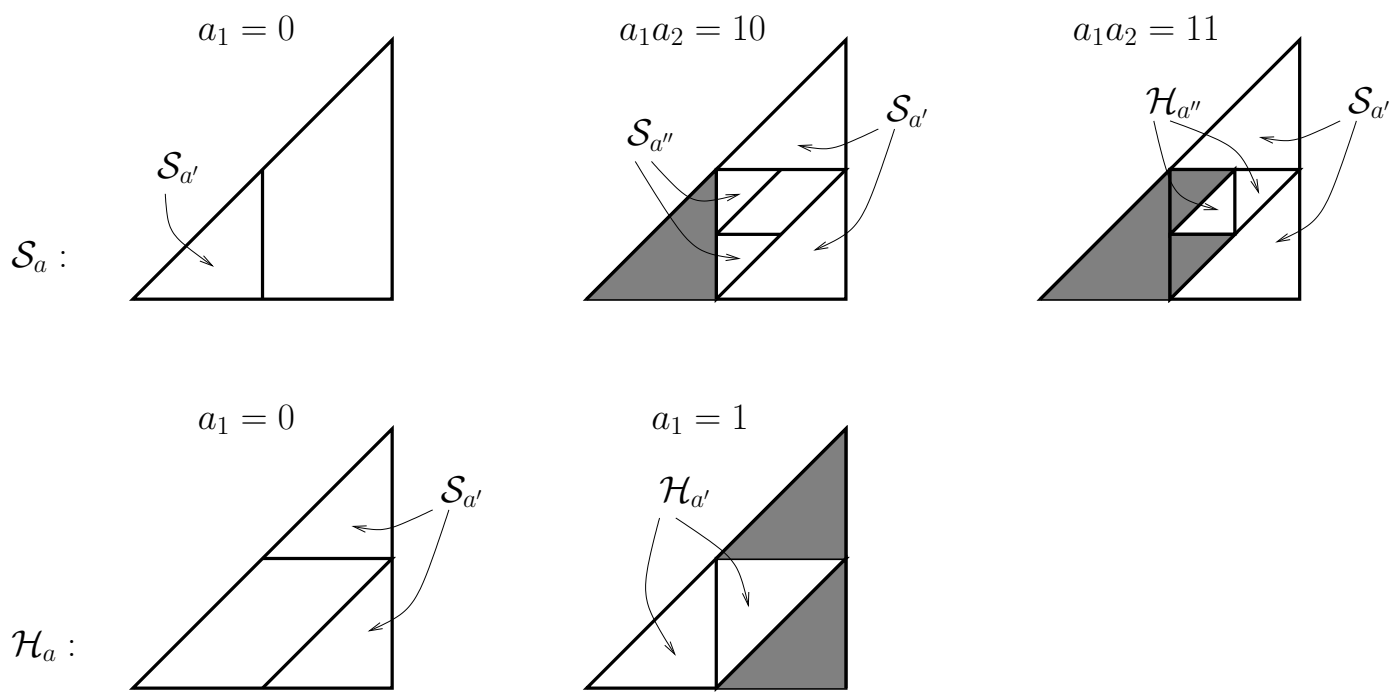

Fig. 7. SHD. Dark areas are fully covered by appropriately mapped $W$, while arrows indicate the placement of suitably transformed sets.

It is clear from the construction that both $\mathcal{S}_{a}$ and $\mathcal{H}_{a}$ are well defined and increasing on dyadic rationals. Moreover, we claim that $|a-b|<2^{-n}$ implies

$$
d_{H}\left(\mathcal{S}_{a}, \mathcal{S}_{b}\right) \leq \sqrt{2} \cdot 2^{-n}, \quad d_{H}\left(\mathcal{H}_{a}, \mathcal{H}_{b}\right) \leq \sqrt{2} \cdot 2^{-n},
$$

where $d_{H}$ is the Hausdorff metric. To prove (7.1), we proceed by induction on $n$. Note that we can assume $a<b$ and, by monotonicity, that $a_{k}=0$, for $k \geq n$ and $b_{n}=1$. The assumption also means that $a_{k}=b_{k}$ for $k<n$. As $\operatorname{diameter}(W)=\operatorname{diameter}(\delta W)=\sqrt{2},(7.1)$ is easy to verify for $n=0$ and $n=1$. For $n \geq 2$, we can use the induction hypothesis on $a^{\prime}, a^{\prime \prime}$ and $b^{\prime}, b^{\prime \prime}$. Note that all $a^{\prime}$ sets are normalized by $\frac{1}{2}$ and all $a^{\prime \prime}$ sets by $\frac{1}{4}$, which yields (7.1).

The uniform continuity (7.1) immediately implies that we can extend both $\mathcal{S}_{a}$ and $\mathcal{H}_{a}$ uniquely to $a \in[0,1]$, so that (7.1) holds for $a, b \in[0,1], \mathcal{S}_{1}=\mathcal{H}_{1}=W$, and

$$
\mathcal{S}_{a}=\cap_{b>a} \mathcal{S}_{b}=\operatorname{closure}\left(\cup_{b<a} \mathcal{S}_{b}\right), \mathcal{H}_{a}=\cap_{b>a} \mathcal{H}_{b}=\operatorname{closure}\left(\cup_{b<a} \mathcal{H}_{b}\right),
$$


where $b$ in all cases ranges over dyadic rationals. The following result identifies $\mathcal{S}_{a}$ as a subsequential limit of $A_{t}$.

Theorem 4. Assume that $t_{n}=a \cdot 2^{n}$, for $a \in[0,1]$ and $A_{0}=\{0\}$. The 12 rules that do not have both $\pi(3)=1$ and $\pi(4)=1$ exhibit subsequential convergence to SHD:

$$
2^{-n} A_{t_{n}} \rightarrow \mathcal{S}_{a}
$$

in the Hausdorff metric, as $n \rightarrow \infty$, uniformly in a. ${ }^{3}$

Proof. For dyadic rationals $a$, the proof follows from arguments in Section 6 . The case of general $a \in[0,1]$ is then a consequence of monotonicity of $A_{t}$ and $\mathcal{S}_{t}$.

The sets $\mathcal{S}_{a}$ are difficult to visualize for $a$ 's that are not dyadic rationals. In particular, it is clear that they have a very complicated boundary. It is therefore natural to ask whether one can determine the fractal (Hausdorff) dimension $\operatorname{dim}_{H}$ of the said boundary. The answer in many cases is yes, and we turn to this task now.

As in [GG1], we need to count the number of buds of size $2^{-n}$. Let us denote this number by $f_{n}(a)$ for the triangle and $g_{n}(a)$ for the (half) hole dynamics. Then

$$
f_{n}(a)= \begin{cases}f_{n-1}\left(a^{\prime}\right) & \text { if } a_{1}=0, \\ 2 f_{n-1}\left(a^{\prime}\right)+2 f_{n-2}\left(a^{\prime \prime}\right) & \text { if } a_{1} a_{2}=10, \\ 2 f_{n-1}\left(a^{\prime}\right)+2 g_{n-2}\left(a^{\prime \prime}\right) & \text { if } a_{1} a_{2}=11,\end{cases}
$$

and

$$
g_{n}(a)= \begin{cases}2 f_{n-1}\left(a^{\prime}\right) & \text { if } a_{1}=0, \\ 2 g_{n-1}\left(a^{\prime}\right) & \text { if } a_{1}=1 .\end{cases}
$$

When $a$ is rational, these equations reduce to a finite linear recursion, so that $f_{n}(a) \sim c \lambda^{n}$, for some $c>0$ and $\lambda \in(0,4]$. If $\lambda \leq 2$, then it follows immediately that $\operatorname{dim}_{H}\left(\partial \mathcal{S}_{a}\right)=1$. If $\lambda>2$, then these are Maudlin-Williams [MW] fractals. Hence the box counting and Hausdorff dimensions agree, and $\operatorname{dim}_{H}\left(\partial \mathcal{S}_{a}\right)=\log \lambda / \log 2$.

To illustrate, for $N \geq 2$ let us take $a$ with periodic binary representation that repeats $N-1$ initial 1's and then a single 0 , so $a=\left(2^{N}-2\right) /\left(2^{N}-1\right)$. Index the shifted configurations with superscripts. We get

$$
g_{n}^{1}=2 g_{n-1}^{2}=4 g_{n-2}^{3}=\cdots=2^{N-2} g_{n-(N-2)}^{N-1}=2^{N-1} f_{n-(N-1)} .
$$

Also,

$$
\begin{aligned}
& f_{n}^{N-1}=f_{n-1}^{N}=f_{n-1}, \\
& f_{n}^{N-2}=2 f_{n-1}^{N-1}+2 f_{n-2}^{N}=4 f_{n-2}, \\
& f_{n}^{N-3}=2 f_{n-1}^{N-2}+2 g_{n-2}^{N-1}=12 f_{n-3} .
\end{aligned}
$$

Recursively thereafter we get $f_{n}^{N-k}=c_{k} f_{n-k}$, using

$$
f_{n}^{N-k}=2 f_{n-1}^{N-(k-1)}+2 g_{n-2}^{N-(k-2)}=\left(2 c_{k-1}+2^{k-1}\right) f_{n-k} .
$$

\footnotetext{
${ }^{3}$ The limit also equals $\rho \mathcal{S}_{a}$ in the weak sense, but Hausdorff convergence seems more to the point.
} 


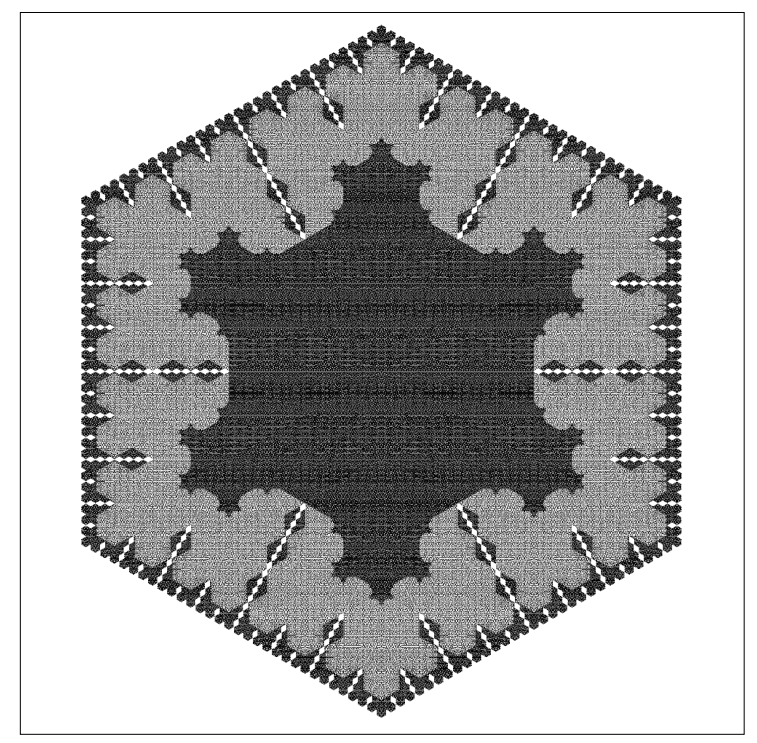

Fig. 8. Occupied set of Hex 13 , started at $\{0\}$, at times $\left\lfloor a \cdot 2^{10}\right\rfloor$ for $a=2 / 3$, $14 / 15$, and $62 / 63$ in darker, lighter, and darker shade, respectively. This illustrates problems with surmising fractal properties from pictures: the $2 / 3$ case appears to have the "most fractal" boundary, which in fact has dimension 1 . The fairly large fractal dimension in the other two cases is a consequence of growth that appears at the exposed corners and is not visible at this scale.

Therefore $c_{1}=1$ and for $k \geq 1, c_{k}$ satisfy the recursion $c_{k}=2 c_{k-1}+2^{k-1}$. It follows that $c_{k}=k \cdot 2^{k-1}$, so $f_{N}=N \cdot 2^{N-1} f_{n-N}$, and $\lambda=2 \cdot(N / 2)^{1 / N}$. Hence

$$
\operatorname{dim}_{H}\left(\partial \mathcal{S}_{a}\right)=1-\frac{1}{N}+\frac{\log _{2} N}{N}
$$

For example, $\operatorname{dim}_{H}\left(\partial \mathcal{S}_{2 / 3}\right)=1, \operatorname{dim}_{H}\left(\partial \mathcal{S}_{14 / 15}\right)=\operatorname{dim}_{H}\left(\partial \mathcal{S}_{245 / 255}\right)=5 / 4$, the dimension achieves its maximum (within this collection of examples) of approximately 1.289 at $a=62 / 63$, and tends to 1 as $N \rightarrow \infty$. See Fig. 8 .

Note that we do not know how to determine $\operatorname{dim}_{H}\left(\mathcal{S}_{a}\right)$ when $a$ is irrational, nor the maximum possible value of $\operatorname{dim}_{H}\left(\mathcal{S}_{a}\right)$.

The Diverse Hole Dynamics (DHD) is associated with the $4 \mathrm{Hex}$ rules that have $\pi(3)=$ $\pi(4)=1$. Besides $\mathcal{S}_{a}$, there are now three hole dynamics $\mathcal{H}_{a}^{i} \subset W, i=1,2,3$, all initialized at $\emptyset$ when $a=0$ (cf. Fig. 9). When $a_{1}=0$ or $a_{1} a_{2}=10$, the recursion for $\mathcal{S}_{a}$ is the same as before, while if $a_{1} a_{2}=11$,

$$
\begin{aligned}
& \mathcal{S}_{a}=\frac{1}{2} W \quad \cup\left(\frac{1}{2}, 0\right)+\frac{1}{2} \mathcal{S}_{a^{\prime}} \quad \cup \quad\left(\frac{1}{2}, 0\right)+\frac{1}{2} \mathcal{S}_{a^{\prime}} \cup\left(\frac{1}{2}, 0\right)+\frac{1}{4} W \\
& \cup\left(\frac{1}{2}, \frac{1}{2}\right)+\frac{1}{4} \rho_{-\pi / 2} \delta W \quad \cup\left(\frac{1}{2}, \frac{1}{4}\right)+\frac{1}{4} \mathcal{H}_{a^{\prime \prime}}^{1} \quad \cup \quad\left(1, \frac{1}{2}\right)+\frac{1}{4} \rho_{\pi} \mathcal{H}_{a^{\prime \prime}}^{3} .
\end{aligned}
$$

If $a_{1}=0$, then

$$
\begin{aligned}
& \mathcal{H}_{a}^{1}=\frac{1}{2} \mathcal{S}_{a}^{\prime} \quad \cup \quad(1,1)+\frac{1}{2} \rho_{\pi} \sigma \mathcal{S}_{a^{\prime}} \quad \cup \quad(1,0)+\frac{1}{2} \rho_{\pi / 2} \delta \mathcal{S}_{a^{\prime}} \\
& \mathcal{H}_{a}^{2}=\mathcal{H}_{a}^{3}=(1,1)+\frac{1}{2} \rho_{\pi} \sigma \mathcal{S}_{a^{\prime}} \quad \cup \quad(1,0)+\frac{1}{2} \rho_{\pi / 2} \delta \mathcal{S}_{a^{\prime}}
\end{aligned}
$$



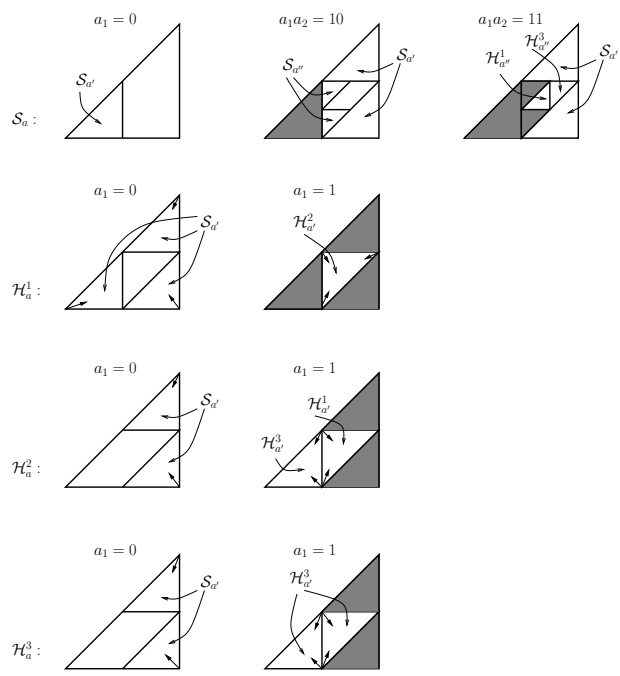

Fig. 9. DHD. Live vertices within the $\mathcal{H}$ 's are indicated by arrows.

while if $a_{1}=1$,

$$
\begin{aligned}
& \mathcal{H}_{a}^{1}=\frac{1}{2} W \quad \cup \quad(1,1)+\frac{1}{2} \rho_{\pi} \sigma W \quad \cup(1,0)+\frac{1}{2} \rho_{\pi / 2} \delta W \quad \cup \quad \frac{1}{2} \rho_{\pi} \mathcal{H}_{a^{\prime}}^{2}, \\
& \mathcal{H}_{a}^{2}=(1,1)+\frac{1}{2} \rho_{\pi} \sigma W \quad \cup \quad(1,0)+\frac{1}{2} \rho_{\pi / 2} \delta W \quad \cup \quad \frac{1}{2} \rho_{\pi} \mathcal{H}_{a^{\prime}}^{1} \cup\left(1, \frac{1}{2}\right)+\frac{1}{2} \rho_{\pi} \mathcal{H}_{a^{\prime}}^{3} \\
& \mathcal{H}_{a}^{3}=(1,1)+\frac{1}{2} \rho_{\pi} \sigma W \quad \cup \quad(1,0)+\frac{1}{2} \rho_{\pi / 2} \delta W \quad \cup \quad \frac{1}{2} \rho_{\pi} \mathcal{H}_{a^{\prime}}^{3} \quad \cup \quad\left(1, \frac{1}{2}\right)+\frac{1}{2} \rho_{\pi} \mathcal{H}_{a^{\prime}}^{3} .
\end{aligned}
$$

These are extended to $a \in[0,1]$ in the same way as before, yielding the following result.

Theorem 5. Assume that $t_{n}=a \cdot 2^{n}$, for $a \in[0,1]$, and $A_{0}=\{0\}$. The 4 rules with $\pi(3)=1$ and $\pi(4)=1$ exhibit convergence to DHD:

$$
2^{-n} A_{t_{n}} \rightarrow \mathcal{S}_{a},
$$

in the Hausdorff metric as $n \rightarrow \infty$, uniformly in a.

It follows from Section 6 that for these 4 rules, most finite seeds give rise to macroscopic dynamics different from DHD. The more general construction is quite analogous, however, so we will not describe it in detail.

Dimension computations are similar to those for SHD, but more complicated. Now there are $f_{n}(a)$ and $g_{n}^{i}(a), i=1,2,3$, that satisfy

$$
f_{n}(a)= \begin{cases}f_{n-1}\left(a^{\prime}\right) & \text { if } a_{1}=0 \\ 2 f_{n-1}\left(a^{\prime}\right)+2 f_{n-2}\left(a^{\prime \prime}\right) & \text { if } a_{1} a_{2}=10 \\ 2 f_{n-1}\left(a^{\prime}\right)+g_{n-2}^{1}\left(a^{\prime \prime}\right)+g_{n-2}^{3}\left(a^{\prime \prime}\right) & \text { if } a_{1} a_{2}=11\end{cases}
$$


and

$$
\begin{aligned}
& g_{n}^{1}(a)= \begin{cases}3 f_{n-1}\left(a^{\prime}\right) & \text { if } a_{1}=0, \\
g_{n-1}^{2}\left(a^{\prime}\right) & \text { if } a_{1}=1,\end{cases} \\
& g_{n}^{2}(a)= \begin{cases}2 f_{n-1}\left(a^{\prime}\right) & \text { if } a_{1}=0, \\
g_{n-1}^{1}\left(a^{\prime}\right)+g_{n-1}^{3}\left(a^{\prime}\right) & \text { if } a_{1}=1,\end{cases} \\
& g_{n}^{3}(a)= \begin{cases}2 f_{n-1}\left(a^{\prime}\right) & \text { if } a_{1}=0, \\
2 g_{n-1}^{3}\left(a^{\prime}\right) & \text { if } a_{1}=1 .\end{cases}
\end{aligned}
$$

For the same example $a=\left(2^{N}-2\right) /\left(2^{N}-1\right)$ as in the SHD case, a similar but more involved computation yields

$$
\operatorname{dim}_{H}\left(\partial \mathcal{S}_{a}\right)=\frac{1}{N} \cdot \log _{2}\left(\frac{1}{3} N \cdot 2^{N}+\frac{7}{9} 2^{N}-\frac{11}{9}-\frac{5}{9} 1_{\{N \text { even }\}}\right) .
$$

\section{Thickness and related issues}

Let us begin the proof of Theorem 3 by considering the 8 digital snowflakes that are not exactly solvable. For the argument that $A_{\infty}$ is never thick, we address only the hardest case, Hex 1456 ; the same method applies equally well to the other 7 rules.
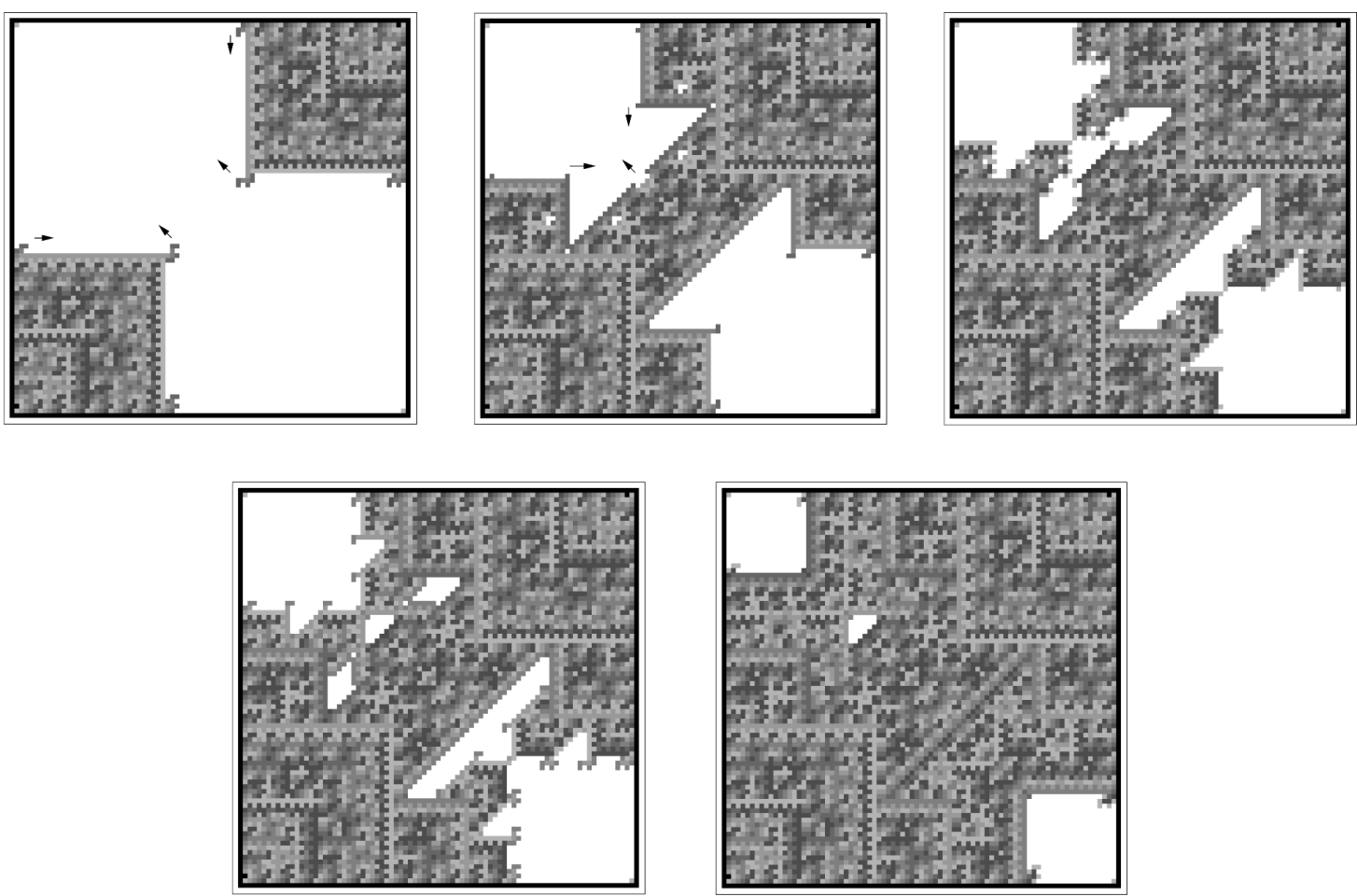

Fig. 10. Dynamics in $H(84)$ at times $34,49,56,58,66$. Arrows indicate which directions of boundary dynamics (from nearby buds) lead to the residual hole.

The key fact (initially suggested by simulations) is that holes of sizes in the sequence below, 
obtained by doubling and subtracting 4 ,

$$
84,164,324,644, \ldots,
$$

create holes, each of which is twice as large as the previous one. Although this should follow from a general rescaling property of the dynamics (which we do not even know how to formulate), the property is a consequence a finite chain of basic interactions, as illustrated by a sequence of intermediate times for $H(84)$ in Fig. 10.

Before we write out the details, note that the configuration in the hole is symmetric with respect to the map $(x, y) \mapsto(L-y, L-x)$, and write $L=2^{n}+2^{n-2}+4, n \geq 6$. The first important configuration to consider occurs at time $t_{1}=2^{n-1}+2\left(t_{1}=34\right.$ when $\left.n=6\right)$. Concentrate on the extreme boundary dynamics generated by two buds: $b_{1}$ at $\left(2,2^{n-1}+4\right)$ (moving eastward) and $b_{2}$ at $\left(2^{n-1}+2,2^{n-1}+4\right)$ (moving diagonally towards the northwest), and their two symmetrically located counterparts $b_{1}^{\prime}$ and $b_{2}^{\prime}$. Now let us pause at the time $t_{2}=t_{1}+2^{n-2}-1$ (49 in the example). The two buds $b_{2}$ and $b_{2}^{\prime}$ would have created two diagonals

$$
\begin{aligned}
& \left(2^{n-1}+2-2^{n-2}+1,2^{n-1}+4\right) \text { to }\left(2^{n-1}+2,2^{n-1}+4+2^{n-2}-1\right) \\
& \left(L-\left(2^{n-1}+4\right)-2^{n-2}+1, L-\left(2^{n-1}+2\right)\right) \text { to }\left(L-\left(2^{n-1}+4\right), L-\left(2^{n-1}+2\right)+2^{n-2}-1\right)
\end{aligned}
$$

if not for the interaction between them. By additivity, we need to eliminate the intersection between the two, which consists of 2 sites, as is easy to check. At the same time, $b_{1}$ creates a column

$$
\left(2^{n-2}+2,2^{n-1}+4\right) \text { to }\left(2^{n-2}+2,2^{n-1}+4+2^{n-2}-1\right) .
$$

Note that this does not interfere with the diagonal created by $b_{2}$ (on which the smallest $x$ coordinate is $\left.2^{n-2}+3\right)$.

Now it is easy to see that the gap of two sites created by the interaction between $b_{2}$ and $b_{2}^{\prime}$ creates two buds which generate the same boundary dynamics (in the northwest direction) as would a single 1 at time $t_{2}-1$ immediately southeast from the gap, at $\left(2^{n-1}+2,2^{n-1}+\right.$ $2^{n-2}+2$ ). Call this seed $b_{3}$. Also consider the bud $b_{4}$ (and its symmetric counterpart $b_{4}^{\prime}$ ) at $\left(2^{n-2}+3,2^{n-1}+2^{n-2}+4\right)$ (immediately northeast from the top of the column in the previous paragraph), which creates extreme boundary dynamics moving eastward. The next time to pause is just before these two interact, at time $t_{3}=t_{2}+2^{n-3}-1$ (which is 56 in our example). At this time, $b_{3}$ creates a diagonal

$$
\left(2^{n-1}+2-\left(2^{n-3}-1\right), 2^{n-1}+2^{n-2}+2\right) \text { to }\left(2^{n-1}+2,2^{n-1}+2^{n-2}+2+2^{n-3}-1\right)
$$

plus two new buds at its ends, one to the west of the bottom point, one to the north of the top point. It follows that the boundary dynamics from $b_{4}$ is free to make one more step free from interruption from $b_{3}$ to create a column

$$
\left(2^{n-1}-2^{n-3}+2,2^{n-1}+2^{n-2}+4\right) \text { to }\left(2^{n-1}-2^{n-3}+2,2^{n-1}+2^{n-2}+4+2^{n-3}-3\right) .
$$

This column would be higher if not for interference from $b_{4}^{\prime}$ which creates a horizontal column at the same time. These two, together with an additional site from $b_{3}$, seal the hole at time $t_{3}+1$. Three new sites are occupied inside at the next time, but the remaining sites remain 0 forever (if $n \geq 6$ ).

Since the dynamics create a hole of every size in the sequence, infinitely many times and no matter what the finite seed $A_{0}$ (cf. Sections 4 and 6), it follows that there are arbitrarily large islands of 0 's in the final state $A_{\infty}$. Therefore $A_{\infty}$ is not thick. 
The argument that $A_{\infty}^{c}$ is always thick for Hex 1 is quite simple. Namely, we will show that every 1 in $A_{\infty}$ that is at $\ell^{\infty}$-distance 3 or more from $A_{0}$ must have a 0 within $\ell^{\infty}$-distance 2 . Assuming this is not the case, let a 1 at $x$ be a counterexample. Let $t$ be the time at which $x$ becomes 1 . We label $0_{x}$ the 0 at $x$ at the time $t-1$. We will also assume, without loss of generality, that the single 1 in $x+\mathcal{N}$ at this time is to the left of $x$.

$\begin{array}{lll} & 0 & 0 \\ 0 & 0_{2} & 0_{1} \\ 0 & 1 & 0_{x} \\ 0 & 0_{1} & \\ 0 & 0 & \end{array}$

Fig. 11. Local configuration at time $t-1$.

It follows that the two 0's labeled $0_{1}$ in Fig. 11 must also become 1 at time $t$ (to avoid being a 0 with two neighboring 1's), and then so does $0_{2}$. This forces the remaining 0's in Fig. 11 and yields a contradiction, as the 1 cannot be isolated from other 1's.

Let us turn next to the exactly solvable rules. To show that $A_{\infty}$ is thick when $A_{0}=\{0\}$, we simply note that the smallest wedges and holes contain 1's, and choose a suitable $R$, an upper bound on the distance between any 0 and the set of 1's. Any easy induction shows that $R$ provides the same bound for larger wedges and holes. General initial sets are handled by arguments in Section 6. (Note, however, that the maximal distance of a 0 from the set of 1 's in $A_{\infty}$ may not be bounded independently of $A_{0}$.) Thickness of $A_{\infty}^{c}$ is equally easy to establish.

To finish the proof of Theorem 3, we need to demonstrate (2).

We start with Hex 13456. First note that a finite seed will generate six rays of 1's via the extreme boundary dynamics. Assume that there is a nonempty connected (in the hexagonal lattice sense) set of 0 's in $A_{\infty}$. If this set is infinite, then it must extend to infinity, say, between the eastern and northeastern ray of 1's. Hereafter we refer to the sites within that portion of $A_{\infty}$. Start at some point between the rays, and move up to the topmost 0 , call it $0_{1}$. Assume first that its northeast neighbor is 0 , call it $0_{2}$. There cannot be any 0 's above $0_{2}$ (or else the topmost 0 would see at least three 1 's). Then the northeast neighbor of $0_{2}$ must be a 0 , call it $0_{3}$, again with no 0's above it. Continuing in this fashion, we create an infinitely extended diagonal of 0's. But the boundary dynamics alone precludes this, for otherwise they would never create a 1 directly above the diagonal, and then directly above that, etc., finally eliminating the ray of 1's. Therefore the right neighbor of $0_{1}$ must be a 0 , again call it $0_{2}$, and the entire column above $0_{2}$ must be 1 's. By the same argument, the right neighbor of $0_{2}$, named $0_{3}$, must be a 0 and the entire column above it 1's. This procedure eventually creates a horizontal ray of 0 's, which is equally impossible by the boundary dynamics. The only remaining possibility is that the connected set of 0 's is finite. But then simply observe that the rightmost among its top 0's must see at least three 1's. This contradiction demonstrates that $A_{\infty}^{c}=\emptyset$, as claimed.

We conclude this section by showing that Hex 13456 is the only case for which $A_{\infty}$ does not have infinitely many 0's regardless of $A_{0}$. It is easy to observe that the other three candidates (i.e., rules with density 1) may leave some 0's: Hex 136 and Hex 1346 will not fill a domino 00 in a sea of 1's, while Hex 1356 will not fill a triangle ${ }_{0}^{00}$ in a sea of 1's.

To generate infinitely many 0's, let the initial set $A_{0}$ consists of a column of $L 1$ 's. Then the primary boundary encloses a triangle with this column as one side, at time $L$. This repeats every time the boundary dynamics recreates the initial set, hence infinitely many times. To show that 
there is an $A_{0}$ with infinitely many 0 's in $A_{\infty}$, it is therefore enough to find an $L$ for which the described triangle does not completely fill in. By exhaustive computer search, the smallest such $L$ 's for rules Hex 136, Hex 1346, and Hex 1356 are, respectively, 5, 19, and 42. We do not know if there are smaller seeds with this property, but it seems that very small random initial seeds (those that fit into $5 \times 5$ box, say) are very likely to have $A_{\infty}=\mathbb{Z}^{2}$.

\section{$9 \quad$ Exact Solvability}

The phrase "exact solvability" is popular in statistical physics [Bax], but its exact meaning is difficult to pin down. By contrast, as we will now explain, in the discrete world of deterministic $\mathrm{CA}$ this concept does have a natural rigorous framework based on computational complexity. We will restrict our formulations to the final set $A_{\infty}$, although generalization to the entire space-time evolution is straightforward. (In fact, at the cost of an extra dimension, it is easy to represent the evolution of any two-state CA as the final configuration of a solidification rule.) Intuitively, we require that "for a given $x \in \mathbb{Z}^{2}$, it is computationally easy to decide whether $x \in A_{\infty}$." This notion of course depends on an initial set $A_{0}$, which will be assumed to be $\{0\}$ unless otherwise specified. Formally, we call a solidification CA exactly solvable (from $A_{0}$ ) if there exists a finite automaton which, upon encountering $x$ as input, decides whether $x \in A_{\infty}$. Representation of $x$ as input is given as $\left( \pm i_{1}^{1}, \pm i_{1}^{2}, i_{2}^{1}, i_{2}^{2}, \ldots\right)$, where $i_{1}^{1}, i_{1}^{2}$ are the most significant binary digits of the first and second coordinate of $x ; i_{2}^{1}, i_{2}^{2}$ the next most significant, etc. (Some initial $i_{k}^{1}$ 's or $i_{k}^{2}$, s may be 0 , and the representation is finite but of arbitrary length.) This means that $A_{\infty}$ is automatic [AS], or equivalently a uniform tag system [Cob]. More general representations of inputs are sometimes desirable, particularly if one wants to study CA on more general lattices, but this one suffices for our present purposes. Note that exact solvability puts a limit on the computational complexity of a CA: for example, it cannot be universal. ${ }^{4}$ Also, by [Cob, Theorem 6] such $A_{\infty}$ cannot have an irrational density, in contrast to the limit set in [GriH] generated by a suitable initial seed in the Game of Life.

To our knowledge, the simplest nontrivial example of an exactly solvable CA is Diamond 1 solidification, which is a modification of Hex 1 using the neighborhood $\{(0,0),(0, \pm 1),( \pm 1,0)\}$. In this case (the "intricate, if very regular, pattern of growth" depicted on p. 171 of [Wol3]), it can be shown by induction that $x \notin A_{\infty}$ iff $\max \left\{k: i_{k}^{1}=1\right\}=\max \left\{k: i_{k}^{2}=1\right\}$. It is easy to construct a (two-state) finite automaton that checks this condition, and the density $\rho$ of $A_{\infty}$ evidently must satisfy the equation $\rho=1 / 2+\rho / 4$, so that $\rho=2 / 3$. It is also worth noting that the first quadrant portion of this $A_{\infty}$ is a fixed point of the substitution system $1 \rightarrow{ }_{11}^{10}, 0 \rightarrow{ }_{01}^{10}$. Although, by Cobham's theorem ([Cob, Theorem 3], [AS, Theorem 14.2.3]), a substitution representation of $A_{\infty}$ must exist in any exactly solvable case, we have no simple explicit construction for our Hex examples.

The proof that Hex 13 is exactly solvable is quite a bit messier than the recursion for $b_{n}$, as symmetry seems difficult to exploit. We now sketch the construction of the required finite automaton, as encoded in Fig. 12. The number of states will be determined by a number of possible division squares. Except in three cases, these squares are further divided into two triangles by the northeast diagonal. Each of these triangles is either a wedge, in which case it is equipped with an arrow at the vertex from which the wedge propagates in the dynamics, or

\footnotetext{
${ }^{4}$ Note that, since there are one-dimensional universal CA [Coo], there are two-dimensional universal solidification CA. A universal CA can generate any recursive language, hence one which is not recognizable by a finite automaton.
} 

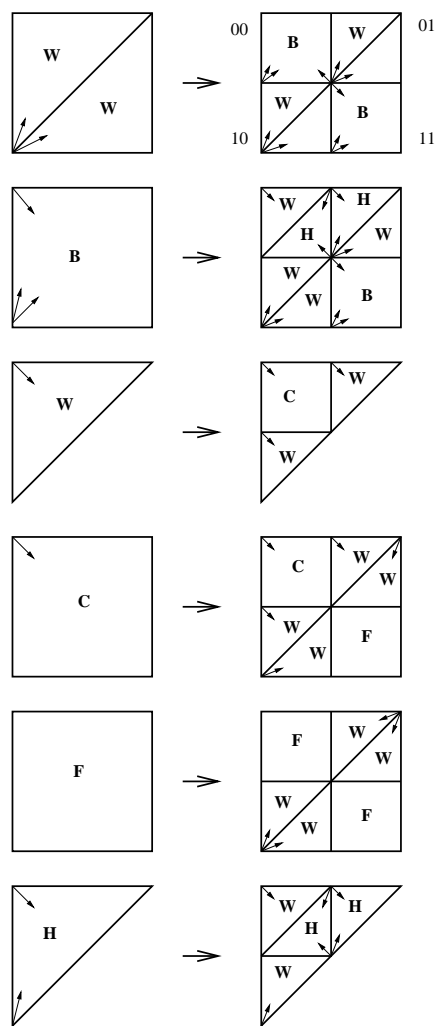

Fig. 12. Division algorithm. Transition according to the value of $i_{1}, i_{2}$ is indicated in the top line, and pieces are labeled in the obvious fashion.

a half hole which is equipped with two arrows. The half hole encodes dynamics filling in one half of the diamond-shaped holes. We need to distinguish wedges and half holes with different orientations, so there are 6 of the former and 4 of the latter. The first exceptional (undivided) division square is a full hole, which has a single representative and encodes filling a square hole. The other two exceptions, arbitrarily named B-square and $C$-square, have four representatives each and encode filling the "complex" part of a wedge. (A B-square is marked by 3 arrows, while a C-square is marked by a single arrow, as shown in Fig. 12.) The number of possible division squares can be reduced by observing that two half-holes cannot appear, and neither can two wedges with arrows which originate at the corners of the southeast diagonal. This makes a total of 29 possible division squares.

Assume that both initial signs are + . Draw the initial division square, representing the initial state of the automaton, which has two wedges, each with an arrow at the lower left corner. The situation is reflected when both the initial signs are - , while in the case of $\mathrm{a}+$ and $\mathrm{a}-$ the initial state is a $\mathrm{C}$-square.

After reading $\left(i_{1}^{1}, i_{1}^{2}\right)$, the next state is one of the 4 subsquares, two of the same type as the initial one, the other two B-holes. This division is of course dictated by how the dynamics fills the square. The next two bits induce further division, and so forth. Fig. 12 provides a check that any of the 29 division squares gets divided into 4 division squares.

A minor final complication is that the holes are not divided into two smaller holes by exact wedges, but by their translation in a coordinate direction by 1 . These corrections do not 
accumulate, as they go in opposite direction from one scale to the next, as illustrated by Fig. 13.

One can also prove that Hex 134 is exactly solvable by the same method. The algorithm is a little more involved, as it necessitates several more types of full and half holes, but there is nothing conceptually new, so we omit the details.

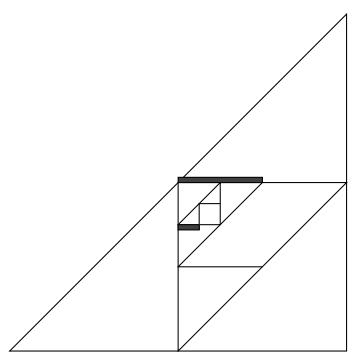

Fig. 13. Hole correction. First correction (on the larger scale) is in the direction $(0,1)$, next in the direction $(0,-1)$.

As it turns out, we have already established enough structure to establish the relative complexity of the 8 remaining digital snowflakes. Indeed, the following result [GraH], together with Theorems $1-3$, proves that none of them can be exactly solvable from any initial set.

Lemma 9.1. If an automatic set $S \subset \mathbb{Z}^{2}$ has a strictly positive asymptotic density, then it is thick.

In conclusion, we remark that complexity analysis of cellular automata by means of finite automata was initiated by S. Wolfram in [Wol2]. The perspective of Wolfram's paper is in a sense opposite to ours, in that it measures how complexity of the set of all possible configurations increases over time. In contrast, we consider a single configuration at the "end of time."

\section{References}

[Ada] J. A. Adam, Flowers of ice-beauty, symmetry, and complexity: a review of "The Snowflake: Winter's Secret Beauty," Notices Amer. Math. Soc. 52 (2005), no. 4, 402-416.

[AS] J.-P. Allouche, J. Shallit, "Automatic Sequences: Theory, Applications, Generalizations," Cambridge University Press, 2003.

[Bax] R. J. Baxter, "Exactly Solved Models in Statistical Mechanics," Academic Press, 1982.

[BCG] E. R. Berlekamp, J. H. Conway, R. K. Guy, "Winning Ways for Your Mathematical Plays," Second Edition, Vol. 4, Chapter 25, Peters 2004.

[BH] W. A. Bentley and W. J. Humphreys, "Snow Crystals," McGraw-Hill, 1931 and Dover, 1962.

[Cob] A. Cobham, Uniform tag sequences, Math. Systems Theory 6 (1972), 164-192.

[Coo] M. Cook, Universality in elementary cellular automata, Complex Systems 15 (2005), $1-40$. 
[Des] R. Descartes, "Les Météores," 1637; éd. Adam et Tannery, Paris, Vrin, t. IV, 1965.

[Dix] R. Dixon, "Mathographics," Dover, 1991.

[Fel] W. Feller, "An Introduction to Probability Theory and Its Applications, Volume 1," Wiley, 1968.

[Gar] M. Gardner, Mathematical games, Scientific American 133 (December 1976), 124-128.

[GG1] J. Gravner, D. Griffeath, Cellular automaton growth on $\mathbb{Z}^{2}$ : theorems, examples and problems, Advances in Applied Mathematics 21 (1998), 241-304.

[GG2] J. Gravner, D. Griffeath, Modeling snow crystal growth II, in preparation.

[GraH] J. Gravner, D. Hickerson, Asymptotic density of an automatic sequence is uniform, in preparation.

[GriH] D. Griffeath, D. Hickerson, A two-dimensional cellular automaton with irrational density. In "New Constructions in Cellular Automata," edited by D. Griffeath and C. Moore, Oxford Univ. Press, 2003.

[Gle] J. Gleick, "Chaos: Making a New Science," Penguin Books, 1987.

[Gri] D. Griffeath, http://psoup.math.wisc.edu/extras/1ormore/1ormore.html.

[Hic] D. Hickerson, personal communication.

[Hoo] R. Hooke, "Micrographia," 1665; Dover, 2003.

[Kep] J. Kepler, "Strena Seu de Nive Sexangula," 1611. Translated as "The Six-Cornered Snowflake," trans. Colin Hardie, Clarendon Press, Oxford, 1966.

[Kin] B. W. King, Snowflake Curves, Math. Teacher 57 (1964), 219-222.

[Koc] H. von Koch, Sur une courbe continue sans tangente, obtenue par une construction géométrique élémentaire, Arkiv för Mathematik, Astronomi och Fysik 1 (1904), 681-702.

[Lev] S. Levy, "Artificial Life: The Quest for a New Creation," Pantheon Books, 1992.

[Lib1] K. Libbrecht, http://www.its.caltech.edu/〜atomic/snowcrystals/

[Lib2] K. Libbrecht, The Physics of Snow Crystals, Reports on Progress in Physics, to appear.

[LR] K. Libbrecht, P. Rasmussen, "The Snowflake: Winter's Secret Beauty." Voyageur Press, 2003.

[Mag] O. Magnus, "De Variis Figuris Nivium," 1555.

[Mea] P. Meakin, "Fractals, Scaling and Growth Far from Equilibrium," Cambridge University Press, 1998.

[MW] R. D. Mauldin, S. C. Williams, Hausdorff dimension in graph directed constructions, Trans. Amer. Math. Soc. 309 (1988), 811-829.

[Nak] U. Nakaya, "Snow Crystals: Natural and Artificial," Harvard University Press, 1954. 
[Pac] N. H. Packard, Lattice models for solidification and aggregation, Institute for Advanced Study preprint, 1984. Reprinted in "Theory and Application of Cellular Automata," S. Wolfram, editor, World Scientific, 1986, pp. 305-310.

[PV] A. Pimpinelli, J. Villain, "Physics of Crystal Growth," Cambridge University Press, 1999.

[Wil] S. J. Willson, Cellular automata can generate fractals, Discrete Appl. Math. 8 (1984), 91-99.

[Woj] M. Wójtowicz, Mirek's Cellebration: a 1D and 2D Cellular Automata explorer. http://www.mirwoj.opus.chelm.pl/ca/

[Wol1] S. Wolfram, Computer software in science and mathematics, Scientific American 251 (September 1984), 188-203.

[Wol2] S. Wolfram, Computation theory of cellular automata, Comm. Math. Phys. 96 (1984), $15-57$.

[Wol3] S. Wolfram, "A New Kind of Science," Wolfram Media, 2002. 